\title{
Strength predictions of clear wood at multiple scales using numerical limit analysis approaches
}

\author{
Mingjing Li1 ${ }^{1, *}$, Josef Füssl ${ }^{1}$, Markus Lukacevic ${ }^{1}$, Josef Eberhardsteiner ${ }^{1}$, Chris M. Martin ${ }^{2}$
}

\begin{abstract}
This work aims at a new approach to understand failure mechanisms and to predict wood strengths, which are strongly influenced by the complex hierarchical material system of wood. Thus, a mechanical concept, where different microstructural characteristics are incorporated, appears to be necessary, based on the division of wood into meaningful scales of observation. At each scale, effective strength properties are to be determined and a multiscale approach is applied, for which conventional numerical methods appear to be inefficient. Thus, within this work, numerical limit analysis approaches are further developed and applied for the first time to wood, complementing conventional methods successfully at certain scales of observation in a multiscale 'damage' concept.

Limit analysis belongs to the group of direct plastic analysis methods, exclusively focusing on the time instant of structural collapse, and delivering the ultimate strength. Compared with conventional numerical approaches, limit analysis approaches are much more stable and efficient.

In this work, orthotropic failure criteria and periodic boundary conditions have been implemented into numerical limit analysis formulations. As numerical results, effective failure surfaces are obtained at both annual ring scale and clear wood scale. A validation at clear wood scale renders this new approach as very promising.
\end{abstract}

Keywords:

strength prediction of wood, numerical limit analysis approaches, different length scales, orthotropic failure criteria, periodic boundary conditions

\section{1. Introduction}

In recent years, wood, as a building material, has undergone a revival, which can be attributed to its excellent mechanical and physical properties on one hand and the fact that it is an environmentally sustainable material with a pleasant appearance on the other hand. And due to continuous extensions and improvements in building codes throughout Europe, allowing higher and more complex timber constructions, its share of the building market is constantly increasing and the volume consumption is facing enormous growth rates. In the course of this, however,

\footnotetext{
*Corresponding author

Email address: mingjing.li@tuwien.ac.at (Mingjing Li)

${ }^{1}$ Vienna University of Technology, Karlsplatz 13/202, Vienna, 1040, Austria.

${ }^{2}$ University of Oxford, Parks Road, Oxford, OX1 3PJ, U.K.
} 
demands on timber constructions are increasing constantly. In order to meet these demands and allow the use of wood in complex applications, prediction tools for the mechanical performance of wood are gaining importance. A wide repertoire of advanced prediction tools should facilitate a better utilisation of wood and wood-based products, increasing their competitiveness compared with other building materials. Especially for predicting the ultimate strength of wood, only very few reliable and promising methods exist so far. A brief overview of the existing methods for predicting/modelling structural failure at the wooden board level is given in the following.

\subsection{Prediction tools for the ultimate strength of wood}

The first group of approaches avoids the direct description of failure mechanisms, and instead uses so-called mean stress concepts Masuda (1988), where averaged stresses over a finite small area are assumed to indicate failure. These areas can be adjusted to typical features of wood, such as structural characteristics of wood fibers Aicher et al. (2002). Serrano and Gustafsson (2007), Sjödin and Serrano (2008) and Sjödin et al. (2008) applied this approach in combination with findings of linear elastic fracture mechanics. They investigated single and multiple dowel connections, where the size of the finite area was governed by the fracture properties of the material. The suitability of different area definitions, over which the stresses are averaged, and also the efficiency of various failure criteria was compared by Guindos (2011). Lukacevic and Füssl (2014) presented a physically-based structural failure criterion, where it was assumed that in wooden boards with knots, global failure can be related to a stress transfer mechanism, which is identifiable by evaluating averaged stress fields in the vicinity of knots. Either way, such models are mostly dependent on empirical parameters and the true failure mechanisms cannot be identified. This can be overcome by directly modelling failure processes.

The most sophisticated approaches for this are based on multi-surface plasticity/failure criteria, as described in Mackenzie-Helnwein et al. (2003) and Schmidt and Kaliske (2006). Thereby, orthotropic yield surfaces are defined describing the onset of ductile failure whereas these surfaces indicate stress states where cracks are to be expected. Cracking is normally modelled with so-called cohesive elements, including an anisotropic traction separation law for wood. Applications of this model show promising results regarding the estimation of load carrying behaviour Schmidt and Kaliske (2007, 2009). Danielsson and Gustafsson (2014) also used a cohesive zone model based on plasticity theory for parameter studies of a glued laminated timber beam with a hole.

These approaches work well for a homogeneous fibre orientation and if the location of the crack path is known in advance. The weak point of these methods is the influence on the failure mechanism by specifying the crack direction. As wood is a naturally grown material, usually complex stress states prevail, especially in the presence of knots and other defects, where such an approach is difficult to apply or additional strategies are required, like the approach in Jenkel and Kaliske (2014), where cohesive interface elements are aligned with predetermined fiber directions around knots.

At the author's institute, Lukacevic and Füssl (2014); Lukacevic et al. (2014a); Lukacevic and Füssl (2016); Lukacevic et al. (2017) have established the basis for a crack initiation and propagation criterion in the framework of the eXtended Finite Element Method (XFEM) in recent years, which has been implemented into a numerical simulation tool for wooden boards. The implementation of such an approach poses two questions: (i) which stress states cause the initiation 
of a crack? and (ii) what is the corresponding crack direction at the wooden-board scale? These questions can only be answered by looking deeper into the microscopic hierarchical structure of wood, and taking several structural features at different length scales into account. Therefore, to obtain reliable failure surfaces and reliable crack directions at the wooden-board scale, a multiscale 'damage' approach is pursued. For such an approach, failure mechanisms at different length scales of wood need to be analysed numerically. Doing this exclusively by applying the concept of multisurface plastic failure criteria in combination with XFEM at each length scale, leads to very high computational cost and to a probably unnecessarily high complexity of the overall model.

For this reason, an additional numerical method is to be introduced, the numerical limit analysis. This method, a so-called 'direct method', exclusively focuses on the time instant of failure, and delivers lower and upper bounds for the ultimate strength of the considered material structure. Compared to conventional approaches, where the complete load history has to be considered and, in order to predict correct failure mechanisms, proper regularisation techniques must be used, the limit analysis approach is much more stable and efficient. Moreover, it leads to rigorous bounds of the material strength and, thus, give a reliable error measure of the prediction. Thus, this method can serve as a useful tool to complement more complex numerical step-by-step approaches by, for example, identifying critical failure regions in a preliminary simulation procedure, as also suggested in Füssl et al. (2017); Pisano et al. (2015).

Of course, these advantages result from strict limitations on which these formulations are based, including: (i) the associated plastic flow rule and (ii) the rigid and perfectly plastic (ductile) material behaviour. For wood, these two limitations are not entirely correct, but, the first can be considered as an appropriate assumption, which is made due to a lack of the information about the non-associativity, and the second does not exclude, that good strength predictions are also possible for strain-softening structures. In Denton and Morley (2000) it is stated: "A structure does not need to exhibit perfect plasticity for the theoretical plastic collapse load based on the peak yield stress of each component to be approached closely. Rather, it is necessary that, at the point when a collapse mechanism forms under a particular loading, all those regions within the structure which are undergoing straining lie very close to the peak yield stress which they can achieve." Wood definitely has the ductile potential to 'activate' the strength in many points along a potential crack surface before the brittle failure occurs. Nevertheless, one might argue that this approach, mainly evolved from and applied in fields dealing with very homogeneous (manmade) materials (like steel), is not suitable for application to wood, where failure is often induced by the largest defect (such as, e.g., knots at the wooden board scale or cell wall imperfections an observation scale below). With regard to this it might be mentioned that the prediction quality of concepts addressing structural failure of a very heterogeneous material heavily depends on knowledge about the local strength reduction due to defects. Thus, tools which are capable of analysing this influence for a huge amount of defect variations within an acceptable timeframe, like numerical limit analysis, might be very useful for fracture models at the macroscale. For this reason, the numerical limit analysis approach seems to be an appropriate method to make a comprehensive multiscale 'damage' approximation for wood worth pursuing. 


\subsection{Numerical limit analysis}

Originally, the objective of limit analysis was the determination of the load bearing capacity of structures exhibiting elastoplastic material response. At collapse, the capacity of structures to store any additional external work as recoverable energy is lost. Thus, for a prescribed macroscopic velocity field and a prescribed macroscopic traction field throughout the boundary, defining the loading situation, limit analysis concentrates on the critical energy dissipation rate at failure of structures or, in this paper, of unit cells for microstructures. The problem may be stated as follows: Find the kinematically admissible velocity field, which minimises the external energy over the set of all statically admissible stress fields, which maximise the internal dissipated energy according to Ciria et al. (2008). Unfortunately, the so-obtained saddle-point problem can be solved exactly only for simple geometric and loading situations, and for simple material behaviour. For more complex situations, the plastic flow compatibility in the static lower bound principle and the plastic admissibility in the kinematic upper bound principle may be relaxed, providing lower and upper bounds for the load bearing capacity (effective strength) of structures.

The first complete formulations of limit analysis theorem was established in 1950s by Drucker et al. (1951, 1952); Hill (1951), in which analytical exact solutions (coincident lower and upper bounds) were limited to very simple problems. Thanks to the rapid evolution of computer technology and the development in mathematical programming, the FEM (finite element method) has proven to be a powerful tool for the limit analysis approach, from simple two-dimensional problems to complicated three-dimensional applications. Therefore, more attention has been drawn to numerical limit analysis formulations within past decades.

The early implementation of the finite element method and the optimisation theory into limit analysis was done by Lysmer (1970) for the lower bound problem, and by Anderheggen and Knöpfel (1972) and Maier et al. (1972) for the upper bound problem. In these works, linear three-noded triangular elements were used for discretisation and the underlying optimisation problem could be solved by linear programming, provided that the used failure criteria were linearised.

Many subsequent works can be found focusing on non-linear optimisation and higher order triangular elements for the discretisation. Notable contributions with respect to the non-linear programming have been given by Lyamin and Sloan (2000, 2002a,b); Krabbenhøft and Damkilde (2000), enabling non-linear failure criteria being formulated in their native form. However, local smoothing of yield surfaces with singularities, like the Tresca or Mohr-Coulomb critera, were necessary.

In general, the efficiency of the numerical limit analysis method significantly depends on the algorithms for solving the associated optimisation problem. In later studies by Makrodimopoulos and Martin (2006, 2007), Ciria et al. (2008), Portioli et al. (2014), and in Füssl et al. (2008) for composite materials, the second-order cone programming (SOCP) has proven to be an excellent alternative method, with sufficient robustness and efficiency to solve large-scale optimisation problems of limit analysis. This method is also applicable to a large variety of failure criteria, since many commonly-used failure functions can be cast as second-order cones. Therefore, within this work, the SOCP is employed for the numerical limit analysis formulation.

\subsection{Objective of the paper}

The main objectives driven this work can be formulated as follows: 
- The formulation of numerical limit analysis approaches so that they are applicable to certain observation scales of wood. Therefore required is the extension of conventional FEM-based limit analysis formulations to incorporate orthotropic failure criteria and periodic boundary conditions.

- The application of these formulations to selected structures of the annual ring scale as well as the clear wood scale to demonstrate the applicability of this method. Moreover, 2D strength information in terms of effective failure surfaces are to be obtained, to show the potential and possible future benefits of such an approach.

- The comparison of the numerically obtained effective failure surfaces at the clear wood scale with experimental results from biaxial testing, to assess the accuracy and capability of the proposed limit analysis concept with respect to the prediction of wood strengths.

The paper is structured as follows: In the next two sections, classical lower bound formulations (Section 2.1) and upper bound formulations (Section 2.2) are recapitulated and the implementation of periodic boundary conditions, for unit cell considerations, as well as orthotropic failure criteria are presented. The application of these newly derived formulations to wood unit cells of the annual ring scale, and the resulting failure modes and effective failure surfaces, are presented in Section 3.1. Subsequently, in Section 3.2, effective failure surfaces at the clear wood scale are obtained and compared to experimental results. Finally, a brief summary and concluding comments are given in Section 4.

\section{FEM formulation of limit analysis}

The lower bound theorem is based on statically admissible stress fields, $\boldsymbol{\sigma}$, satisfying the equilibrium equations, the failure criterion and static boundary conditions in each point of the considered structure, while the upper bound theorem is built up on kinematically admissible velocity fields, $\dot{\mathbf{u}}$, fulfilling the compatibility equation, the plastic flow rule and kinematic boundary conditions over the whole structure. Neither of them necessarily gives the exact load multiplier $\beta^{*}$ for structural failure, but they provide a lower bound $\beta^{L B}$ and an upper bound $\beta^{U B}$, fulfilling

$$
\beta^{L B} \leq \beta^{*} \leq \beta^{U B}
$$

When the so-called bracketing error, defined as the difference between $\beta^{L B}$ and $\beta^{U B}$, is smaller than the required accuracy of the prediction, a sufficient approximation of $\beta^{*}$ is obtained, which represents a complete solution satisfying the equilibrium equation, the compatibility equation, and the failure function with associated flow.

In this paper, both lower and upper bound formulations are developed in 2D and applied to $2 \mathrm{D}$ unit cells representing microstructures of wood at different scales of observation. Note that, at each scale, the characteristic dimensions of the unit cells in the $2 \mathrm{D}$ plane is much smaller than the dimension of the material in the out-of-plane direction. Therefore, the plane strain condition is enforced in all of following formulations. 


\subsection{Lower bound formulation}

The lower bound theorem states that any load multiplier determined from a statically admissible stress field which is in equilibrium with given external loads is not greater than the exact collapse multiplier $\beta^{*}$. Therefore, for the given discretisation, the best lower bound of the load multiplier, $\beta^{L B}$, can be found through the following optimisation problem:

$$
\begin{array}{lll}
\beta^{L B}=\max & \beta \\
\text { s.t. } & \mathbf{L}_{\sigma} \boldsymbol{\sigma}+\beta \mathbf{h}=\mathbf{0} & \text { in } V \\
& \mathbf{L}_{t} \boldsymbol{\sigma}=\beta \mathbf{t} & \text { on } \Gamma_{t} \\
& f(\boldsymbol{\sigma}) \leq 0 & \text { in } V
\end{array}
$$

where in the first constraint, enforcing equilibrium within the body $V, \mathbf{L}_{\sigma}$ denotes the divergence operator and $\mathbf{h}$ the live body force vector. In the static boundary conditions, $\mathbf{L}_{t}$ is the tractionstress operator depending on the outward normal vector $\mathbf{n}$ of the loaded surface $\Gamma_{t}$ and $\mathbf{t}$ represents the prescribed live traction forces. Plastic admissibility, defined by the yield function $f$, is enforced by the third constraint.

For the discretisation of the stress field $\boldsymbol{\sigma}$ triangular elements with three nodes are used, in which the stress field is approximated with linear shape functions. Each element has its own set of internal stress evaluation nodes, thus multiple nodes from adjacent elements may share the same coordinates. At discontinuities between elements, the continuity of normal and shear stress components is enforced. The discretised version of the optimisation problem Eq. (2) and its adaptions for periodic boundary conditions and orthotropic failure criteria are described step by step in the following.

\subsubsection{Equilibrium within elements}

The discretised form of the internal equilibrium, first constraint in Eq. (2), can be expressed for an element as follows:

$$
\begin{aligned}
\mathbf{L}_{\sigma}^{e} \mathbf{N}_{\sigma}^{e} \mathbf{q}_{\sigma}^{e} & +\beta \mathbf{h}^{e}=\mathbf{0}, \quad \forall e \in\{1, \cdots, L E\}, \quad \text { with } \\
\mathbf{L}_{\sigma}^{e} & =\left(\begin{array}{ccc}
\frac{\partial}{\partial x} & 0 & \frac{\partial}{\partial y} \\
0 & \frac{\partial}{\partial y} & \frac{\partial}{\partial x}
\end{array}\right) \in \mathfrak{R}^{2 \times 3}, \quad \text { and } \\
\mathbf{h}^{e} & =\left(\begin{array}{c}
h_{x}^{e} \\
h_{y}^{e}
\end{array}\right) \in \mathfrak{R}^{2},
\end{aligned}
$$

denoting the divergence operator and the body force vector for an element. In this work, no dead and live body forces are taken into account, hence, $\mathbf{h}^{e}=\mathbf{0} . L E$ is the total number of elements, $\mathbf{N}_{\sigma}^{e} \in \mathfrak{R}^{3 \times 9}$ contains linear shape functions interpolating between the nodal stresses $\mathbf{q}_{\sigma}^{e} \in \mathfrak{R}^{9}$, containing all three nodal stress vectors $\mathbf{q}_{\sigma}^{e, i}=\left(q_{\sigma, x x}^{e, i}, q_{\sigma, y y}^{e, i}, q_{\sigma, x y}^{e, i}\right)^{\top} \in \mathfrak{R}^{3}(\forall i \in\{1,2,3\})$ of an element. 


\subsubsection{Equilibrium between elements}

Due to the approximation of the stress field within an element with linear shape functions, the continuity of the stress field between elements can be ensured by applying constraints only to two node pairs of each discontinuity (between elements). Denoting an arbitrary discontinuity as dis with two pairs of adjacent stress nodes designated $\left(1,1^{\prime}\right)$ and $\left(2,2^{\prime}\right)$, the constraints can be expressed as

$$
\left(\begin{array}{cccc}
\mathbf{L}_{t}^{d i s} & \mathbf{0} & -\mathbf{L}_{t}^{d i s} & \mathbf{0} \\
0 & \mathbf{L}_{t}^{d i s} & \mathbf{0} & -\mathbf{L}_{t}^{d i s}
\end{array}\right)\left(\begin{array}{l}
\mathbf{q}_{\sigma}^{d i s, 1} \\
\mathbf{q}_{\sigma}^{d i s, 2} \\
\mathbf{q}_{\sigma}^{d i s, 1^{\prime}} \\
\mathbf{q}_{\sigma}^{d i s, 2^{\prime}}
\end{array}\right)=\mathbf{0}, \quad \forall d i s \in\{1, \cdots, L D\}, \quad \text { with }
$$

$$
\mathbf{L}_{t}^{d i s}=\left(\begin{array}{ccc}
\cos \theta^{d i s} & 0 & \sin \theta^{d i s} \\
0 & \sin \theta^{d i s} & \cos \theta^{d i s}
\end{array}\right) \in \mathfrak{R}^{2 \times 3}
$$

as the $2 \mathrm{D}$ rotation operator with $\theta^{\text {dis }}$ denoting the angle between the discontinuity dis and the $x$-axis of the global coordinate system. $\mathbf{q}_{\sigma}^{d i s, i}=\left(q_{\sigma, x x}^{d i s, i}, q_{\sigma, y y}^{d i s, i}, q_{\sigma, x y}^{d i s, i}\right)^{\top} \in \mathfrak{R}^{3}\left(\forall i \in\left\{1,2,1^{\prime}, 2^{\prime}\right\}\right)$ are the nodal stress vectors associated with the corresponding discontinuity, and $L D$ is the total number of discontinuities of the discretised body.

\subsubsection{Periodic boundary conditions}

Since we want to investigate unit cells of microstructures of wood, the conventional static boundary conditions, appearing in the second constraint in Eq. (2), must be replaced by periodic boundary conditions. Therefore, the relevant portion of the boundary $\partial \Omega$ of each unit cell is divided into two parts, a positive part $\partial \Omega^{+}$and a negative part $\partial \Omega^{-}$with corresponding nodes $\mathbf{x}^{+} \in \partial \Omega^{+}$, $\mathbf{x}^{-} \in \partial \Omega^{-}$, associated outward normal vectors $\mathbf{n}^{+}=-\mathbf{n}^{-}$and the relation $\partial \Omega^{+} \cup \partial \Omega^{-}=\partial \Omega$. In the lower bound formulation, periodicity of the stress field is then ensured by enforcing an anti-periodic traction field through

$$
\mathbf{t}^{+}=-\mathbf{t}^{-},
$$

where $\mathbf{t}^{+}$are traction vectors associated with $\mathbf{x}^{+}$and $\mathbf{t}^{-}$are traction vectors associated with $\mathbf{x}^{-}$. It allows for a periodic succession of the unit cell, and leads to stress fields in the unit cell representative of the whole material structure. Moreover, stress fluctuations vanish by volume averaging over stresses within the unit cell (abbreviated as UC in the following equations) and a meaningful effective (macroscopic) stress $\boldsymbol{\Sigma}$ can be obtained as

$$
\Sigma=\frac{1}{V_{U C}} \int_{V_{U C}} \boldsymbol{\sigma}(\mathbf{x}) \mathrm{d} V
$$

The discretised form of the periodic traction constraint for one pair of corresponding stress nodes, reads

$$
\left(\begin{array}{ll}
\mathbf{L}_{t}^{p e r} & -\mathbf{L}_{t}^{p e r}
\end{array}\right)\left(\begin{array}{c}
\mathbf{q}_{\sigma}^{p e r, \mathbf{x}^{+}} \\
\mathbf{q}_{\sigma}^{\text {per }, \mathbf{x}^{-}}
\end{array}\right)=\mathbf{0}, \quad \forall p e r \in\{1, \cdots, L P\},
$$

where $L P$ is the number of node pairs for which the periodic boundary conditions are defined, $\mathbf{L}_{t}^{p e r} \in \mathfrak{R}^{2 \times 3}$ is identical to the discontinuity operator $\mathbf{L}_{t}^{\text {dis }}$, while $\mathbf{q}_{\sigma}^{\text {per }, \mathbf{x}^{+}}$and $\mathbf{q}_{\sigma}^{\text {per }, \mathbf{x}^{-}}$are the nodal stress vectors for the corresponding node pairs. 
It is then sufficient to apply the external loading through the traction at the surface nodes $\mathbf{x}^{+}$. To define an arbitrary 2D effective loading state the following two sets of constraints, specifying the normal traction components in $x$ and $y$ directions, are necessary:

$$
\begin{array}{ll}
T_{n, x}^{\mathbf{x}^{+}}=\frac{1}{l_{x}} \sum^{L P} \frac{l_{L B}^{p e r}}{2} \mathbf{l}_{t, x}^{p e r} \mathbf{\top} \mathbf{q}_{\sigma}^{p e r, \mathbf{x}^{+}}=\beta q_{t, x}, & \forall p e r \in\{1, \cdots, L P\} \\
T_{n, y}^{\mathbf{x}^{+}}=\frac{1}{l_{y}} \sum^{L P} \frac{l_{L B}^{p e r}}{2} \mathbf{l}_{t, y}^{p e r} \mathbf{\top} \mathbf{q}_{\sigma}^{p e r, \mathbf{x}^{+}}=\beta q_{t, y}, & \forall p e r \in\{1, \cdots, L P\}
\end{array}
$$

with

$$
\left(\begin{array}{c}
q_{t, x} \\
q_{t, y}
\end{array}\right)=\mathbf{q}_{t} \in \mathfrak{R}^{2} \quad \text { and } \quad\left(\begin{array}{l}
\mathbf{l}_{t, x}^{\text {per } \mathbf{T}} \\
\mathbf{l}_{t, y}^{\text {per }} \mathbf{\top}
\end{array}\right)=\mathbf{L}_{t}^{\text {per }},
$$

where $\mathbf{l}_{t, x}^{\text {per }} \in \mathfrak{R}^{3}$ and $\mathbf{l}_{t, y}^{\text {per }} \in \mathfrak{R}^{3}$ are the vectors which map the stress vector $\mathbf{q}_{\sigma}^{\text {per }, \mathbf{x}^{+}}$into the surface traction components $q_{t, x}^{p e r, \mathbf{x}^{+}}, q_{t, y}^{p e r, \mathbf{x}^{+}}$of the node $\mathbf{x}^{+}$in $x$ and $y$ directions, respectively. $l_{L B}^{\text {per }}$ is the element boundary length to which the corresponding node $\mathbf{x}^{+}$belongs, $l_{x}$ and $l_{y}$ are the total lengths of the boundaries in $x$ and $y$ directions, and $q_{t, x}$ and $q_{t, y}$ are prescribed loading factors associated with the macroscopic tractions $\mathbf{T}_{n, x}^{\mathbf{x}^{+}}$and $\mathbf{T}_{n, y}^{\mathbf{x}^{+}}$, respectively.

\subsubsection{Failure criteria}

At the wood cell level, both isotropic and anisotropic plastic behaviour of material phases is encountered. Therefore, two failure criteria have been employed, the orthotropic Tsai-Wu failure criterion Tsai (1965); Tsai and Wu (1971), reading

$$
F_{1} \sigma_{x^{\prime} x^{\prime}}+F_{2} \sigma_{y^{\prime} y^{\prime}}+P_{11} \sigma_{x^{\prime} x^{\prime}}^{2}+P_{22} \sigma_{y^{\prime} y^{\prime}}^{2}+P_{66} \tau_{x^{\prime} y^{\prime}}^{2}+2 P_{12} \sigma_{x^{\prime} x^{\prime}} \sigma_{y^{\prime} y^{\prime}} \leq 1
$$

where $F_{i}(\forall i \in 1,2)$ and $P_{i j}(\forall i, j \in 1,2,3)$ denote strength parameters of the material, and the von Mises isotropic failure criterion, reading

$$
\left(\sigma_{x x}-\sigma_{y y}\right)^{2}+4 \tau_{x y}^{2} \leq \frac{4}{3} k^{2}
$$

where $k$ represents the yield strength of the material. Note that for orthotropic materials, stress variables $\boldsymbol{\sigma}^{\prime}=\left(\sigma_{x^{\prime} x^{\prime}}, \sigma_{y^{\prime} y^{\prime}}, \tau_{x^{\prime} y^{\prime}}\right)^{\top} \in \mathfrak{R}^{3}$ in the failure function Eq. (12) are defined with respect to the local $x^{\prime}-y^{\prime}$ coordinate system in order to take the local principal material orientation into consideration. For isotropic material, no local principal material orientation is required, and the failure function Eq. (13) can be expressed in the global coordinates $x$ and $y$. Since both failure functions, Eq. (12) and Eq. (13), are quadratic, they can be transformed into the general form,

$$
\mathbf{F}^{\top} \boldsymbol{\sigma}^{\prime}+\boldsymbol{\sigma}^{\prime \top} \mathbf{P} \boldsymbol{\sigma}^{\prime} \leq 1
$$

${ }^{242}$ where $\mathbf{P} \in \mathfrak{R}^{3 \times 3}$ and $\mathbf{F} \in \mathfrak{R}^{3}$ contain material strength parameters, and having the form

$$
\mathbf{F}=\left(\begin{array}{c}
F_{1} \\
F_{2} \\
F_{6}
\end{array}\right), \quad \mathbf{P}=\left(\begin{array}{ccc}
P_{11} & P_{12} & 0 \\
P_{21} & P_{22} & 0 \\
0 & 0 & P_{66}
\end{array}\right)
$$


for the orthotropic Tsai-Wu failure criterion Eq. (12), and

$$
\mathbf{F}=\left(\begin{array}{l}
0 \\
0 \\
0
\end{array}\right), \quad \mathbf{P}=\frac{1}{k^{2}}\left(\begin{array}{ccc}
1 & -1 & 0 \\
-1 & 1 & 0 \\
0 & 0 & 3
\end{array}\right)
$$

for the von Mises failure criterion Eq. (13). Note that, for the latter failure criterion is isotropic and, therefore, the local stress vector is consistent with the global one $\boldsymbol{\sigma}^{\prime}=\boldsymbol{\sigma}$. When the matrix $\mathbf{P}$ is positive definite or semidefinite symmetric, it can be decomposed as $\mathbf{P}=\mathbf{D}^{\top} \mathbf{D}$ with $\mathbf{D} \in \mathfrak{R}^{3 \times 3}$. Then the general form of the yield criterion Eq. (14) can be reformulated into a linear constraint and a second-order cone (SOC) constraint, as follows

$$
\begin{aligned}
& \mathbf{s}=\mathbf{a}+\mathbf{B} \boldsymbol{\sigma}^{\prime} \\
& \mathbf{s} \in \mathscr{C}
\end{aligned}
$$

with

$$
\mathbf{a}=\left(\begin{array}{lllll}
1 & 0 & 0 & 0 & 0
\end{array}\right)^{\top} \in \mathfrak{R}^{5} \quad \text { and } \quad \mathbf{B}=\left(\begin{array}{c}
-\mathbf{F}^{\top} / 2 \\
\mathbf{D} \\
\mathbf{F}^{\top} / 2
\end{array}\right) \in \mathfrak{R}^{5 \times 3} .
$$

The definition of the cone set $\mathscr{C}$ and a brief introduction to SOCP is given in Appendix A. Based on Eq. (17) and Eq. (18), the third set of constraints in Eq. (2) can now be written in discretised form as

$$
\begin{array}{ll}
\mathbf{s}^{e, i}=\mathbf{a}^{e}+\mathbf{B}^{e} \mathbf{q}_{\sigma}^{e, i} & \forall e \in\{1, \cdots, L E\} \\
\mathbf{s}^{e, i} \in \mathscr{C}_{e, i} & \forall i \in\{1,2,3\}
\end{array}
$$

with

$$
\mathbf{q}_{\sigma}^{\prime e, i}=\mathbf{R}_{\sigma}^{e} \mathbf{q}_{\sigma}^{e, i}, \quad \text { where } \quad \mathbf{R}_{\sigma}^{e}=\left(\begin{array}{ccc}
\left(\cos \theta^{e}\right)^{2} & \left(\sin \theta^{e}\right)^{2} & -2 \cos \theta^{e} \sin \theta^{e} \\
\left(\sin \theta^{e}\right)^{2} & \left(\cos \theta^{e}\right)^{2} & 2 \cos \theta^{e} \sin \theta^{e} \\
\cos \theta^{e} \sin \theta^{e} & -\cos \theta^{e} \sin \theta^{e} & \left(\cos \theta^{e}\right)^{2}-\left(\sin \theta^{e}\right)^{2}
\end{array}\right) \in \mathfrak{R}^{3 \times 3} .
$$

$\theta^{e}$ specifies the rotation of the local coordinate system with respect to the global one and $\mathbf{q}_{\sigma}^{\prime e, i} \in \mathfrak{R}^{3}$ is the nodal stress vector with respect to the local coordinate system, obtained through the rotation operator $\mathbf{R}_{\sigma}^{e}$.

\subsubsection{Discretized lower bound optimization problem}

Assembling the coefficients of the constraints for the whole discretised body of Eq. (3) in matrix $\mathbf{A}_{L B}^{e q} \in \mathfrak{R}^{2 L E \times 9 L E}$, of Eq. (5) in matrix $\mathbf{A}_{L B}^{d i s} \in \mathfrak{R}^{4 L D \times 9 L E}$, of Eq. (9) in matrix $\mathbf{A}_{L B}^{\text {per }} \in \mathfrak{R}^{2 L P \times 9 L E}$, of Eq. (10) in matrix $\mathbf{B}_{L B}^{\text {per }} \in \mathfrak{R}^{2 \times 9 L E}$, and the SOC constraints according to Eq. (19), leads to the final form of the lower bound optimisation problem:

$$
\begin{array}{ll}
\beta^{L B}=\max & \beta \\
\text { s.t. } & \mathbf{A}_{L B}^{e q} \mathbf{q}_{\sigma}=\mathbf{0} \\
& \mathbf{A}_{L B}^{d i s} \mathbf{q}_{\sigma}=\mathbf{0} \\
& \mathbf{A}_{L B}^{p e r} \mathbf{q}_{\sigma}=\mathbf{0} \\
& \mathbf{B}_{L B}^{p e r} \mathbf{q}_{\sigma}=\beta \mathbf{q}_{t} \\
& \mathbf{q}_{\sigma}^{\prime}=\mathbf{R}_{\sigma} \mathbf{q}_{\sigma} \\
& \mathbf{s}^{s o c}=\mathbf{a}^{s o c}+\mathbf{B}^{s o c} \mathbf{q}_{\sigma}^{\prime} \\
& \mathbf{s}^{e, i} \in \mathscr{C}_{e, i}
\end{array}
$$


with $\forall e \in\{1, \cdots, L E\}, \forall i \in\{1,2,3\}, \mathbf{R}_{\sigma} \in \mathfrak{R}^{9 L E \times 9 L E}, \mathbf{s}^{s o c} \in \mathfrak{R}^{15 L E}, \mathbf{a}^{s o c} \in \mathfrak{R}^{15 L E}$, and $\mathbf{B}^{s o c} \in \mathfrak{R}^{15 L E \times 9 L E}$. Furthermore, $\mathbf{q}_{\sigma} \in \mathfrak{R}^{9 L E}$ and $\mathbf{q}_{\sigma}^{\prime} \in \mathfrak{R}^{9 L E}$ assemble all the nodal stress vectors throughout the body with respect to the global and local coordinate systems, respectively.

\subsection{Upper bound formulation}

The upper bound theorem states that any external load multiplier determined when the internal work rate $W_{\text {int }}$ under a kinematically admissible velocity field $\dot{\mathbf{u}}$ equals the external work rate $W_{\text {ext }}$, is not less than the exact load multiplier $\beta^{*}$. The rate of internal work/dissipation for a body $\Omega$ reads

$$
W_{\text {int }}(\boldsymbol{\sigma}, \dot{\mathbf{u}})=\int_{\Omega} \boldsymbol{\sigma}^{\top} \dot{\boldsymbol{\varepsilon}}(\dot{\mathbf{u}}) \mathrm{d} V,
$$

where $\dot{\boldsymbol{\varepsilon}}(\dot{\mathbf{u}}) \in \mathfrak{R}^{3}$ denotes the $2 \mathrm{D}$ strain rate field. The rate of external work provided by body forces $\mathbf{h}$ and surface traction $\mathbf{t}$ is given by

$$
W_{e x t}(\dot{\mathbf{u}})=\int_{\Omega} \mathbf{h}^{\top} \dot{\mathbf{u}} \mathrm{d} V+\int_{\Gamma_{t}} \mathbf{t}^{\top} \dot{\mathbf{u}} \mathrm{d} A,
$$

but no body force is considered within this work, thus, $\mathbf{h}=\mathbf{0}$. Then, the lowest and therefore the best upper bound load multiplier, $\beta^{U B}$, can be found through the following optimisation problem:

$$
\begin{array}{cll}
\beta^{U B}=\min & W_{\text {int }} & \\
\text { s.t. } & \dot{\boldsymbol{\varepsilon}}=\mathbf{L}_{\dot{u}} \dot{\mathbf{u}} & \text { in } V \\
& \dot{\boldsymbol{\varepsilon}}=\dot{\lambda} \frac{\partial f}{\partial \boldsymbol{\sigma}} & \text { in } V \\
& \dot{\mathbf{u}}=\dot{\mathbf{u}}_{0} & \text { on } \Gamma_{u} \\
& W_{\text {ext }}(\dot{\mathbf{u}})=1 & \text { in } V
\end{array}
$$

where $\mathbf{L}_{\dot{u}} \in \mathfrak{R}^{3 \times 2}$ is the linear compatibility operator, linking the velocity with the strain rate field. The second constraint enforces associated plastic flow throughout the whole body, with $\dot{\lambda}$ as a nonnegative plastic multiplier specifying the magnitude of plastic strain rate, and $f$ denotes the yield function. The kinematic boundary conditions prescribing the velocities $\dot{\mathbf{u}}_{0}$ are represented by the third constraint, and the last constraint simply normalises the external work rate.

The dual of the optimisation problem Eq. (24) can be derived as, according to Makrodimopoulos and Martin (2007),

$$
\begin{array}{lll}
\beta^{U B}= & \max & W_{\text {ext }} \\
\text { s.t. } & \int_{\Omega}\left(\mathbf{L}_{\dot{u}} \dot{\mathbf{u}}\right)^{\top} \boldsymbol{\sigma} \mathrm{d} V=\int_{\Omega} \dot{\mathbf{u}}^{\top} \beta \mathbf{p} \mathrm{d} V & \text { in } V \\
& f(\boldsymbol{\sigma}) \leq 0 & \text { in } V
\end{array}
$$

in which $\mathbf{p}$ denotes the equivalent nodal loading, arising from the body forces $\mathbf{h}$ and the surface tractions $\mathbf{t}$. For the discretisation of the upper bound optimisation problem, 6-node triangular linear-strain elements are used, which are capable of providing very accurate results, as shown in Makrodimopoulos and Martin $(2007,2008)$. Quadratic shape functions for the interpolation of the unknown velocity field are used in Makrodimopoulos and Martin (2005a). According to Makrodimopoulos and Martin (2005b), in the case of unstructured meshes, linear strain elements 
show better performance than constant strain elements with velocity discontinuities. If the vertices of the linear-strain elements are taken as flow-rule points, the obtained solutions are strict upper bounds on the exact collapse load, since the plastic flow rule is enforced to hold throughout the whole element. The stress field in the dual problem has a linear stress distribution within each element. The discretisation of the optimisation problems Eq. (24) and Eq. (25), and their adaptions for periodic boundary conditions and orthotropic plasticity, are shown in the following sections.

\subsubsection{Compatibility between velocity and strain-rate field}

One basic assumption of limit analysis is that all changes in geometry of the body occuring during collapse are negligible, thus, the compatibility between the velocity and the strain-rate field can be expressed by the following linear relation:

$$
\mathbf{q}_{\dot{\varepsilon}}^{e}=\mathbf{A}_{U B}^{e} \mathbf{q}_{\dot{u}}^{e} \quad \forall e \in\{1, \cdots, U E\},
$$

where the vector $\mathbf{q}_{\dot{u}}^{e} \in \mathfrak{R}^{12}$ contains all six nodal velocity vectors $\mathbf{q}_{\dot{u}}^{e, i}=\left(q_{\dot{u}, x}^{e, i}, q_{\dot{u}, y}^{e, i}\right) \in \mathfrak{R}^{2}(\forall i \in$ $\{1, \cdots, 6\})$ of the element, the vector $\mathbf{q}_{\dot{\varepsilon}}^{e} \in \mathfrak{R}^{9}$ contains three nodal strain vectors $\mathbf{q}_{\dot{\varepsilon}}^{e, i} \in \mathfrak{R}^{3}(\forall i \in$ $\{1,2,3\})$ of the element, and

$$
\mathbf{A}_{U B}^{e}=\mathbf{N}_{\sigma}^{e \top} \mathbf{L}_{\dot{u}}^{e} \mathbf{N}_{\dot{u}}^{e} \in \mathfrak{R}^{9 \times 12} \quad \forall e \in\{1, \cdots, U E\}
$$

with

$$
\mathbf{L}_{\dot{u}}^{e}=\left(\begin{array}{ccc}
\frac{\partial}{\partial x} & 0 & \frac{\partial}{\partial y} \\
0 & \frac{\partial}{\partial y} & \frac{\partial}{\partial x}
\end{array}\right) \in \mathfrak{R}^{2 \times 3},
$$

where $\mathbf{N}_{\sigma}^{e} \in \mathfrak{R}^{3 \times 9}$ contains the linear shape functions for the stress field, $\mathbf{N}_{\dot{u}}^{e} \in \mathfrak{R}^{2 \times 12}$ is the quadratic interpolation matrix for the velocity field, and $U E$ is the total number of elements in the upper bound formulation.

\subsubsection{Periodic boundary conditions}

In contrast to the lower bound formulation, where an anti-periodic traction field is enforced over the boundary of the unit cell, in the upper bound formulation a periodic velocity field must be ensured. Therefore, the kinematic boundary constraint in Eq. (24) should be replaced by the following relation:

$$
\dot{\mathbf{u}}^{+}=\dot{\mathbf{u}}^{-}+\dot{\mathbf{E}}\left(\mathbf{x}^{+}-\mathbf{x}^{-}\right),
$$

with $\dot{\mathbf{u}}^{+}$as the velocity vector at the surface node $\mathbf{x}^{+}$, associated with the surface part $\partial \Omega^{+}$, and $\dot{\mathbf{u}}^{-}$as the velocity vector at the surface node $\mathbf{x}^{-}$, associated with the opposite surface part $\partial \Omega^{-}$. $\dot{\mathbf{E}}$ denotes the effective/macrosopic strain rate tensor of the considered unit cell, and is linked to the microscopic strain rate field as follows:

$$
\dot{\mathbf{E}}=\frac{1}{V_{U C}} \int_{V_{U C}} \dot{\varepsilon}(\mathbf{x}) \mathrm{d} V .
$$

Considering the periodic boundary conditions Eq. (7) and Eq. (29), the rate of external energy dissipation, Eq. (23), can be written as,

$$
\begin{aligned}
W_{e x t} & =\int_{\partial \Omega^{+}} \dot{\mathbf{u}}^{+\top} \mathbf{t}^{+} \mathrm{d} S+\int_{\partial \Omega^{-}} \dot{\mathbf{u}}^{-\top} \mathbf{t}^{-} \mathrm{d} S \\
& =\int_{\partial \Omega^{+}} \dot{\mathbf{u}}^{+\top} \mathbf{t}^{+}+\left(\dot{\mathbf{u}}^{+}-\dot{\mathbf{E}} \mathbf{l}\right)^{\top}\left(-\mathbf{t}^{+}\right) \mathrm{d} S=\int_{\partial \Omega^{+}}(\dot{\mathbf{E}} \mathbf{l})^{\top} \mathbf{t}^{+} \mathrm{d} S
\end{aligned}
$$


where $\mathbf{l}$ defines the distance between corresponding node pairs $\left(\mathbf{x}^{+}-\mathbf{x}^{-}\right)$.

After discretization the periodic constraint in Eq. (29) reads

$$
\mathbf{L}_{\dot{u}}^{p e r} \mathbf{q}_{\dot{u}}^{\text {per }}=\dot{\mathbf{E}}^{p e r} \mathbf{1}^{\text {per }} \quad \forall p e r \in\{1, \cdots, U P\},
$$

where $U P$ is the number of node pairs on which periodic boundary conditions are applied, $\dot{\mathbf{E}}^{\text {per }} \in$ $\mathfrak{R}^{2 \times 2}$ is again the effective strain rate tensor of the unit cell, $\mathbf{l}^{\text {per }} \in \mathfrak{R}^{2}$ is the node pair distance of the node pair per, while $\mathbf{q}_{\dot{u}}^{\text {per }}=\left[\mathbf{q}_{\dot{i}}^{\text {per }, \mathbf{x}^{+}}, \mathbf{q}_{\dot{i}}^{p e r, \mathbf{x}^{-}}\right]^{\top} \in \mathfrak{R}^{4}$ contains nodal velocity vectors to which periodic boundary conditions are applied, and $\mathbf{L}_{\dot{u}}^{\text {per }}=[\mathbf{I},-\mathbf{I}] \in \mathfrak{R}^{2 \times 4}$ is the operator linking velocity pairs, with $\mathbf{I} \in \mathfrak{R}^{2 \times 2}$ as the identity matrix.

In the dual form of the optimisation problem, see Eq. (25), the periodic boundary constraint appear in the following expression:

$$
\sum_{e=1}^{U E} \frac{A^{e}}{3}\left(\mathbf{L}_{\dot{u}}^{e} \mathbf{N}_{\dot{u}}^{e} \mathbf{q}_{\dot{u}}^{e}\right)^{\top} \mathbf{N}_{\sigma}^{e} \mathbf{q}_{\sigma}^{e}=\sum_{p e r=1}^{U P}\left(\mathbf{L}_{\dot{u}}^{p e r} \mathbf{q}_{\dot{u}}^{p e r}\right)^{\top} \mathbf{q}_{p}^{p e r}
$$

where $A^{e}$ is the area of the triangle element $e$, and $\mathbf{q}_{p}^{p e r}=\left[\mathbf{q}_{p}^{p e r, \mathbf{x}^{+}}, \mathbf{q}_{p}^{p e r, \mathbf{x}^{-}}\right]^{\top} \in \mathfrak{R}^{4}$ is the equivalent nodal loading vector for the node pair $\mathbf{x}^{+}$and $\mathbf{x}^{-}$.

\subsubsection{Plastic flow rule}

It has been discussed by Makrodimopoulos (2010) that, when the failure function can be cast as a SOC, the set of admissible plastic strains (satisfying the associated flow rull) has the shape of the dual cone. As a result, the plastic flow in Eq. (24) can be reformulated into the form of a SOC as,

$$
\mathbf{e} \in \mathscr{C}^{*}
$$

where $\mathscr{C}^{*}$ is the dual cone of $\mathscr{C}$, which is defined in Eq. (17).

In order to obtain a strict upper bound, both the primal and dual formulations of the upper bound problem are required to be strictly feasible, and, therefore, the constraint of the duality gap,

$$
\mathbf{e}^{\top} \mathbf{S}=0
$$

is required to be satisfied throughout the whole body, according toAndersen et al. (2003).

Considering Eq. (17), the duality gap constraint in Eq. (35) can be expressed as

$$
\mathbf{e}^{\top} \mathbf{s}=\mathbf{e}^{\top} \mathbf{a}+\mathbf{e}^{\top} \mathbf{B} \boldsymbol{\sigma}^{\prime}=0
$$

in which the vector a corresponds to the yield stress and the vector $\mathbf{e}$ is related to the rate of the plastic multiplier $\dot{\lambda}$. Therefore, the internal energy dissipation can be expressed as

$$
W_{i n t}=\int_{\Omega} \mathbf{e}^{\top} \mathbf{a} \mathrm{d} \Omega=\int_{\Omega} \dot{\boldsymbol{\varepsilon}}^{p \top} \boldsymbol{\sigma} \mathrm{d} \Omega,
$$

leading together with Eq. (36) to

$$
\dot{\varepsilon}^{p}=-\mathbf{B}^{\top} \mathbf{e},
$$


as the transformation of the strain rate $\dot{\varepsilon}^{p}$ into the form of a SOC. Since the failure function in Eq. (17) is defined with respect to the local coordinate system, the plastic strain rate in Eq. (38) is also locally defined.

Then, the discretised formulation of the second constraint for the plastic flow rule in Eq. (24) can be expressed as

$$
\begin{aligned}
\mathbf{q}_{\dot{\varepsilon}}^{\prime e, i} & =-\mathbf{B}^{e \top} \mathbf{e}^{e, i} \\
\mathbf{e}^{e, i} & \in \mathscr{C}_{e, i}^{*}
\end{aligned} \quad e \in\{1, \cdots, U E\} \text { and } i=\{1,2,3\}
$$

with

$$
\mathbf{q}_{\dot{\varepsilon}}^{\prime e, i}=\mathbf{R}_{\dot{\varepsilon}}^{e} \mathbf{q}_{\dot{\varepsilon}}^{e, i} \quad e \in\{1, \cdots, U E\} \text { and } i \in\{1,2,3\}
$$

as the nodal strain rate tensor with respect to the local coordinate system. The nodal strain transformation matrix $\mathbf{R}_{\dot{\varepsilon}}^{e}$ is equal to the nodal stress transformation matrix $\mathbf{R}_{\sigma}^{e}$ in Eq. (20).

\subsubsection{Discretised upper bound optimisation problem}

Assembling the coefficients of the compatibility constraints Eq. (26) for the whole discretised body in matrix $\mathbf{A}_{U B}^{c o m} \in \mathfrak{R}^{9 U E \times 2 U N}$, the periodic boundary conditions Eq. (32) in matrix $\mathbf{A}_{U B}^{\text {per }} \in$ $\mathfrak{R}^{U P \times 2 U N}$ and $\dot{\mathbf{E}}_{U B} \mathbf{l}_{U B} \in \mathfrak{R}^{U P}$, and the plastic flow rule constraints Eq. (39) by means of vector $\mathbf{B}^{\text {soc }} \in \mathfrak{R}^{3 U E}$, the primal version of the upper bound optimisation problem, Eq. (24), reads in discretised form:

$$
\begin{aligned}
\beta^{U B}=\min & W_{i n t} \\
\text { s.t. } & \mathbf{A}_{U B}^{c o m} \mathbf{q}_{\dot{u}}=\mathbf{q}_{\dot{\varepsilon}} \\
& \mathbf{A}_{U B}^{p e r} \mathbf{q}_{\dot{u}}=\dot{\mathbf{E}}_{U B} \mathbf{l}_{U B} \\
& \mathbf{q}_{\dot{\varepsilon}}^{\prime}=\mathbf{R}_{\dot{\varepsilon}} \mathbf{q}_{\dot{\varepsilon}} \\
& \mathbf{q}_{\dot{\varepsilon}}^{\prime}=-\mathbf{B}^{s o c \top} \mathbf{e}^{s o c} \\
& \mathbf{e}^{e, i} \in \mathscr{C}_{e, i}^{*}
\end{aligned}
$$

where $e \in\{1, \cdots, U E\}$ and $i \in\{1,2,3\}$. The vectors $\mathbf{q}_{\dot{u}} \in \mathfrak{R}^{2 U E}, \mathbf{q}_{\dot{\varepsilon}} \in \mathfrak{R}^{3 U E}$, and $\mathbf{q}_{\dot{\varepsilon}}^{\prime} \in \mathfrak{R}^{3 U E}$ denote the assembled vectors of the nodal velocities, the nodal strain rates related to the global coordinate system and the nodal strain rates related to the local coordinate system, respectively.

Introducing the definition

$$
\hat{\mathbf{x}}^{e}=\left\{\frac{A^{e}}{3} \mathbf{x}^{e}\right\}_{e=1, \cdots, U E}
$$

where $\mathbf{x}^{e}$ represents an arbitrary position vector of the element $e$, the dual version of the optimisation problem Eq. (41) can be derived from Eq. (25), reading

$$
\begin{aligned}
\beta^{U B}=\max & W_{e x t} \\
\text { s.t. } & \mathbf{A}_{U B}^{c o m} \hat{\mathbf{q}}_{\sigma}=\mathbf{A}_{U B}^{p e r \top} \mathbf{q}_{p} \\
& \hat{\mathbf{s}}=\hat{\mathbf{a}}+\mathbf{B}^{s o c} \hat{\mathbf{q}}_{\sigma}^{\prime} \\
& \hat{\mathbf{q}}_{\sigma}^{\prime}=\mathbf{R}_{\sigma} \hat{\mathbf{q}}_{\sigma} \\
& \hat{\mathbf{s}}^{e, i} \in \mathscr{C}_{e, i}
\end{aligned}
$$

where $e \in\{1, \cdots, U E\}$ and $i \in\{1, \cdots, 6\}$. 
The objective functions in the primal upper bound formulation Eq. (41) and the dual upper bound formulation Eq. (43), respectively, can be defined as follows:

$$
\begin{aligned}
& W_{i n t}=\left(\hat{\mathbf{a}}^{s o c}\right)^{\top} \mathbf{e}^{s o c} \quad \text { and } \\
& W_{e x t}=\left(\dot{\mathbf{E}}_{U B} \mathbf{l}_{U B}\right)^{\top} \mathbf{q}_{p}
\end{aligned}
$$

representing the rates of internal dissipation and external work, respectively.

\section{Application to wood}

An increasingly used and promising mechanistic approach is the so-called multiscale description of material behavior, based on the framework of micromechanics, where a material is modeled at different scales of observation (Ghosh et al., 2001; Michel et al., 1999; Hautefeuille et al., 2012; Saavedra Flores et al., 2016). In recent years, such a model has been developed for wood in Hofstetter et al. (2005, 2007, 2008); Bader et al. (2011); Lukacevic et al. (2014b); Lukacevic and Füssl (2016); Lukacevic et al. (2017) and is currently in elaboration at the author's institute. Until now, this model has mainly been developed for the prediction of effective elastic material properties. The extension to strength predictions represents the next big challenge, and the limit analysis formulations presented above are expected to play a major role in this development.

In this paper, the limit analysis formulations have been applied to selected observation scales as illustrated in Figure 1. It shows a flawless (without knots and other defects) wooden log at the macroscopic scale with the adapted cylindrical coordinate system for the longitudinal $(L)$, radial $(R)$ and tangential $(T)$ directions, taking into account the orthotropy of wood at this scale. Further, the layered structure of earlywood and latewood, so-called annual rings, represents the next scale of observation. On a closer look at these layers, a repetitive honeycomb-like structure is revealed, and the morphological characteristics of which have been extensively described, inter alia, in Fengel and Wegener (1983); Kollmann (1951); Gloimüller et al. (2012). A simplified idealisation of this significantly repetitive structure is illustrated in Figure 1, denoted as annual ring scale. This structure can be suitably represented by two unit cells, shown on the right side of Figure 1. Failure behaviours of these cells strongly influence the failure modes at all higher observation scales. For this reason, the main focus of this paper will be to analyse failure modes of these cell structures by means of numerical limit analysis. This is explained in more detail in following subsections. According to the multiscale approach used in Lukacevic et al. (2014a); Milani et al. (2006a,b), failure stress states and corresponding failure modes at the annual ring scale are obtained, which serve as input to the next higher scale, the clear wood scale. Due to the fact that the clear wood structure also exhibits repetitiveness, it is also approximated by unit cells with periodic boundary conditions. Then, resulting effective failure surfaces are compared to existing experimental data from biaxial-testing published in Eberhardsteiner (1995), as verification for the presented numerical limit analysis method. In this reference, the biaxial test was performed on specimens made of Norway spruce clear wood, thus in this work all unit cell geometries and strength parameters are determined with respect to this wood species.

All computations presented in the following were performed on a Linux desktop machine, with an AMD Phenom(tm) II X6 1090T CPU (6 cores) and 8GB of RAM. While Abaqus is used for 


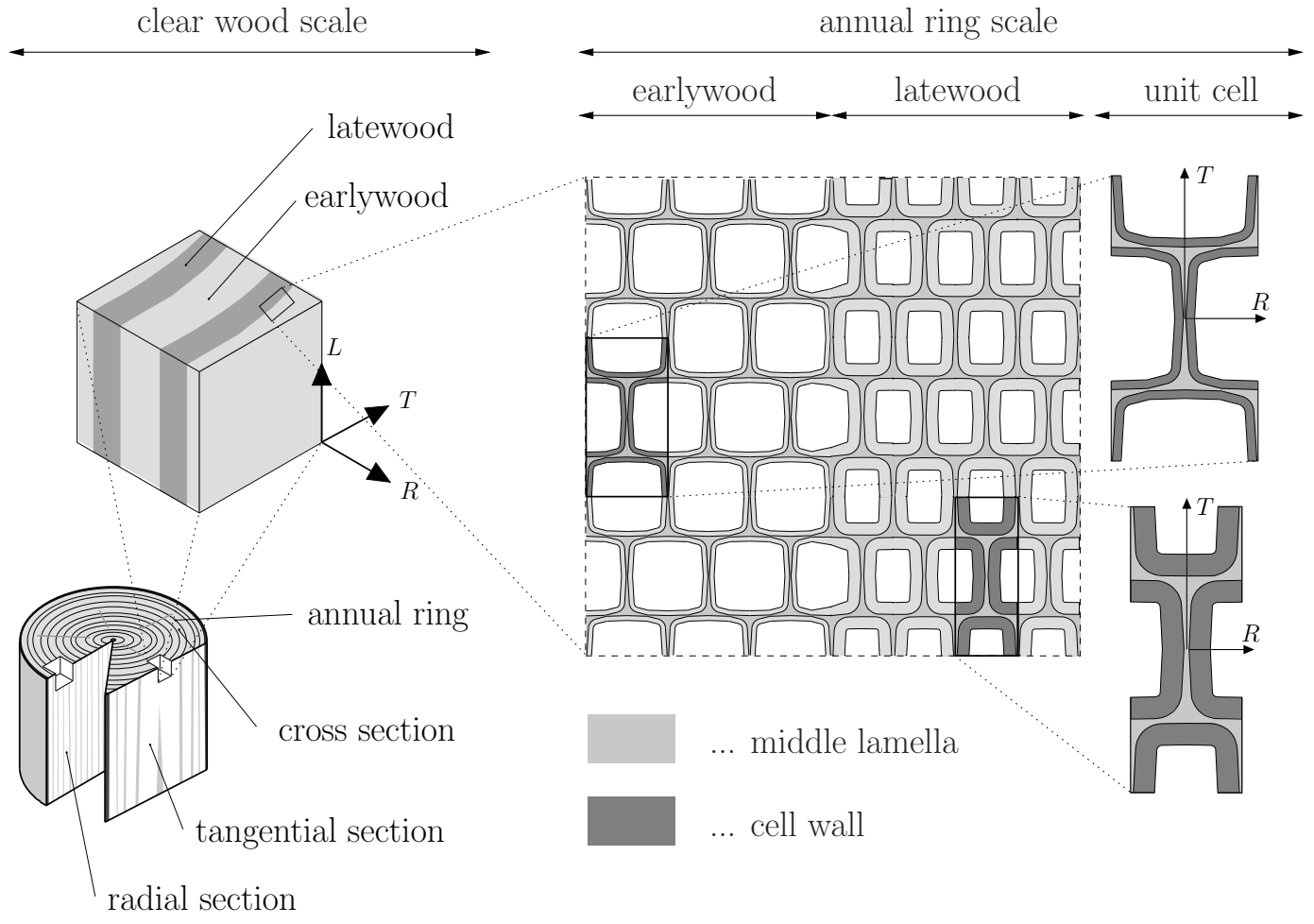

Figure 1: The considered hierarchical structure of wood: The clear wood scale (left) and the annual ring scale (right), where earlywood and latewood unit cells are extracted from the honeycomb-like structure.

the mesh generation and the illustration of results, all other pre- and post-processing tasks as well as the assembly of SOC optimisation problems are carried out by self-written codes in Fortran. Special focus was laid on sparse-matrix manipulations to ensure high efficiency with respect to the variable size and the computational effort. The SOC optimisation problems themselves are solved by the commercial solver MOSEK ApS (2014), which is based on the conic interior-point algorithm developed by Andersen et al. (2003).

\subsection{Application of limit analysis at the annual ring scale}

In the following, the presented limit analysis formulations are applied to the two spruce unit cells illustrated in Figure 1, representing two different types of wood cell at the annual ring scale. The overall mechanical behaviour of each unit cell is dominated by two different material layers, the middle lamella and the cell wall layer. The middle lamella is nearly free of cellulose and, thus, exhibits isotropic behaviour. In contrast, the cell wall layer, dominated by S2 cell wall material, mainly consists of cellulose microfibrils aligning in a certain dominant direction and, thus, exhibits significantly anisotropic behaviour as discussed by Gindl et al. (2004); Gloimüller et al. (2012); Kollmann (1951); Salmen and Burgert (2009). The anisotropic material phase of wood has great similarity with fibre-reinforced composites, and thus, can be appropriately described by an orthotropic Tsai-Wu failure criterion.

The exact geometries of earlywood and latewood cells are adopted from Lukacevic et al. (2014b), 
where they were chosen according to microscopy images and values found in literature for spruce. The earlywood unit cell is discretised with 25196 triangular elements and the latewood unit cell with 25022 . This discretisation and the local material directions $(r$ and $t)$ in the cell wall layer are shown schematically for the earlywood unit cell in Figure 2. To all elements in a certain cell wall layer section, as shown in Figure 2, the same local coordinate system is assigned. In these sections the global stress definition $\left(\sigma_{R R}, \sigma_{T T}, \tau_{R T}\right)^{\top}$ is replaced by the local ones $\left(\sigma_{r r}, \sigma_{t t}, \tau_{r t}\right)^{\top}$.

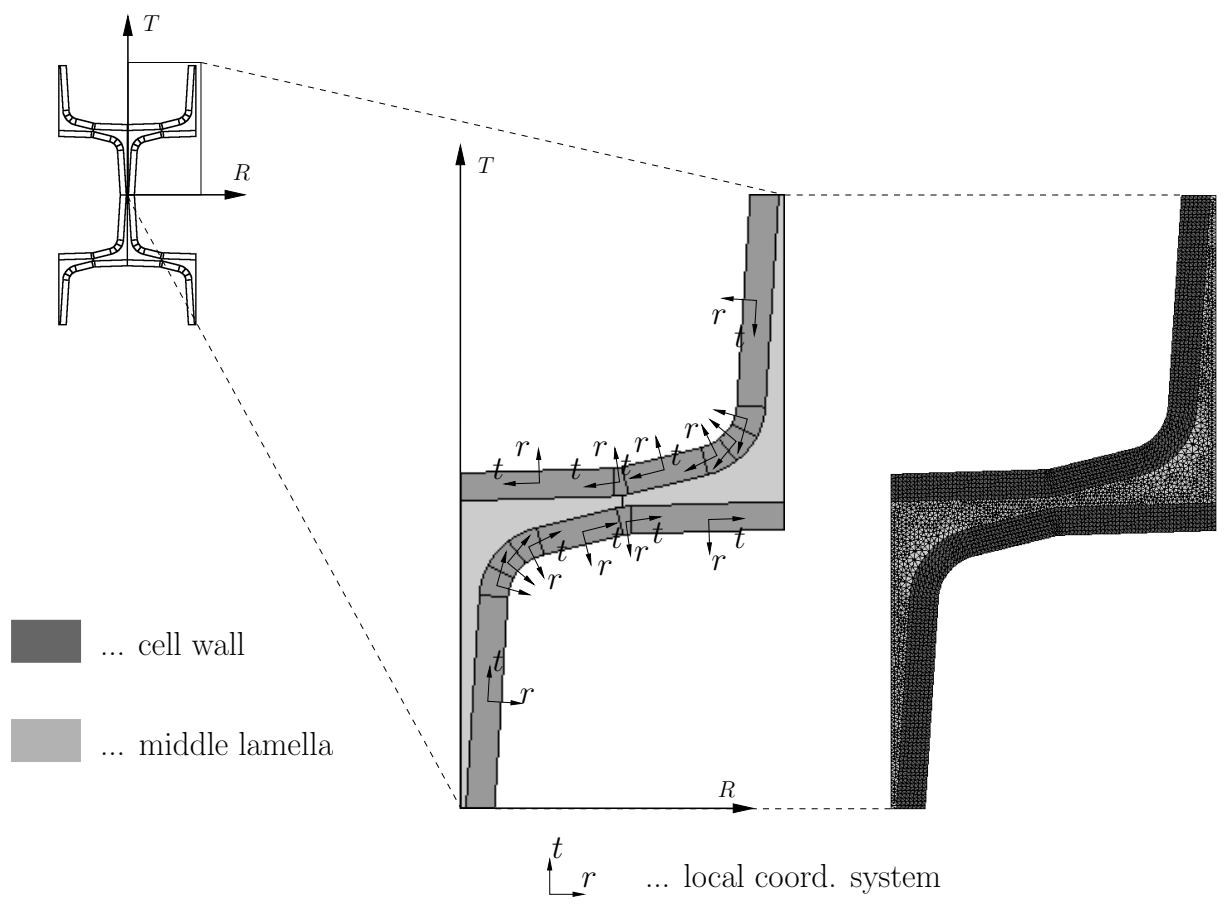

Figure 2: Local material directions in the cell wall layer of an earlywood cell and the corresponding discretisation of such a cell.

Strength parameters assigned to these two material layers are determined on the basis of values found in Bader et al. (2010); Schwiedrzik et al. (2016); Lukacevic et al. (2014b). For the orthotropic Tsai-Wu failure criterion (Eq. (15)), describing the plastic behaviour of the cell wall, they are:

$$
\begin{aligned}
F_{1} & =0 \mathrm{MPa}^{-1} & F_{2} & =0 \mathrm{MPa}^{-1} \\
P_{11} & =4.444 \mathrm{E}-5 \mathrm{MPa}^{-2} & P_{22} & =1.778 \mathrm{E}-4 \mathrm{MPa}^{-2} \\
P_{66} & =5.408 \mathrm{E}-4 \mathrm{MPa}^{-2} & P_{12} & =0 \mathrm{MPa}^{-2}
\end{aligned}
$$

and $k=14.3 \mathrm{MPa}$ is used for the von Mises failure criterion (as shown in Eq. (16)) assigned to the middle lamella. The corresponding failure surfaces are plotted in Figure 3, with respect to the local coordinates $(r$ and $t)$.

Then, the lower bound formulation Eq. (21) and the dual upper bound formulation Eq. (43) are applied to these wood unit cell models, delivering effective strengths and failure mechanisms under various loading situations. A lower bound and an upper bound result for the earlywood unit cell under tangential tensile loading are illustrated in Figure 4. The required computing time for each 


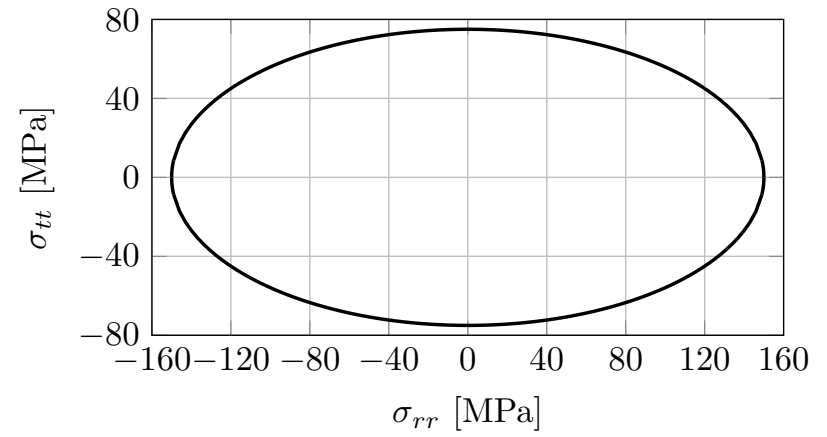

(a)

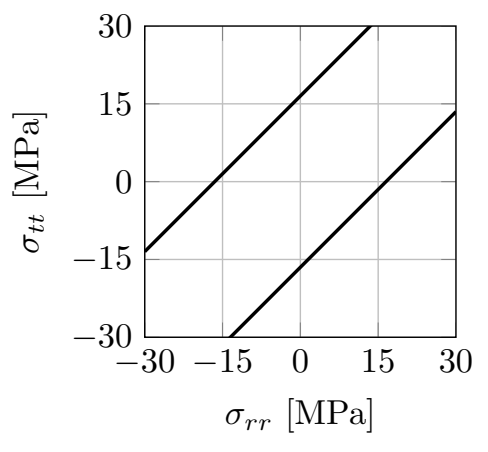

(b)

Figure 3: Constitutive failure surfaces assigned to (a) the cell wall layers and (b) the middle lamella.

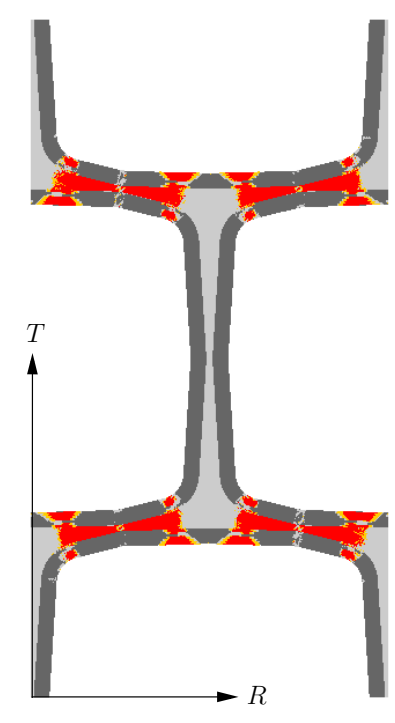

(a)

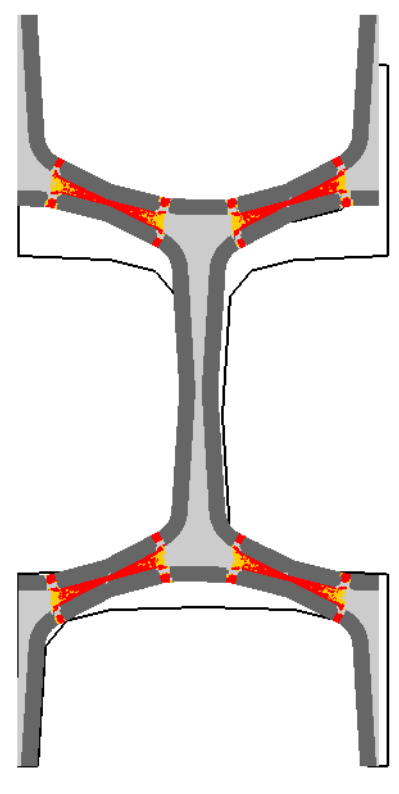

(b)

Figure 4: Illustrative (a) lower bound failure region with the threshold of the objective function value $[-3 E-2: 0]$ and (b) upper bound failure region with the threshold $[-2 E-4: 0]$ (red-coloured areas) for the earlywood unit cell under prescribed tensile loading in the $T$ direction.

of these two simulations was below 2 minutes. In Figure 4, dark and light grey areas indicate the cell wall and the middle lamella, respectively, and the red coloured areas indicate plastic failure. Since in the lower bound formulation only the stress field is considered, no deformation is available from the primal optimization problem and thus none is displayed in Figure 4a. The deformed 
shape in Figure 4b represents the nodal velocity field obtained from the upper bound calculation, whereas the black outline shows the undeformed shape.

The corresponding effective stress states, in this case effective tensile stress states in the $T$ direction, are $\boldsymbol{\Sigma}^{L B}=[0.0,2.43,0.0]^{\top}$ from the lower bound and $\boldsymbol{\Sigma}^{U B}=[0.0,2.47,0.0]^{\top}$ from the upper bound simulations. The resulting bracketing error, defined as $\left.\left(\Sigma_{T}^{U B}-\Sigma_{T}^{L B}\right) / \Sigma_{T}^{U B}+\Sigma_{T}^{L B}\right)=$ $0.81 \%$, is very small. Also taking into account the good agreement between the plastic failure zones of the lower and upper bound results (see Figure 4), the first numerical example of this method can be regarded as promising.

\subsubsection{Determination of effective failure surfaces}

By applying various loading situations, traction forces in the lower bound formulation and material flow through the boundaries in the upper bound formulation, lower and upper bounds for different effective stress states $\boldsymbol{\Sigma}$ and the corresponding failure mechanisms are obtained. Each individual effective stress state represents one point on the effective failure surface of the considered wood unit cell.

For the earlywood unit cell, 116 individual lower bound simulations with a total computing time of 171.2 min and 54 upper bound simulations with 71.2 min computing time were carried out. The resulting lower and upper bounds of the effective failure surface is shown in Figure 5 a. The corresponding lower and upper bound for the latewood unit cell is displayed in Figure 5b, obtained from 116 and 69 lower and upper bound simulations with computing times of 237.4 min and $144.3 \mathrm{~min}$, respectively.

For each unit cell, the lower and upper bound results lead to an almost identical shape and orientation of the effective failure surface. Moreover, for most of the results the bracketing error is quite small. The general difference in the magnitude of the obtained effective strengths in the $R T$-plane between earlywood and latewood cells seems to be realistic, though unfortunately, no experimental data exists at this scale of observation which could be used for validation.

\subsubsection{Failure mechanisms of wood cells}

In a next step, the failure mechanisms associated with the effective failure stress states are analysed, and transitions between failure modes are discussed. For this evaluation it is sufficient to focus on upper bound results, since lower and upper bound simulations provide similar failure modes under similar loading situations, as is shown by the illustrative example in Figure 4.

\section{Earlywood}

In Figure 6, the effective upper bound failure surface of the earlywood unit cell is plotted once again, including a black velocity vector at each stress point, pointing in the direction of the related effective plastic strain rate. It is interesting to note that the associated plasticity of the underlying cell wall layers remains well preserved at this next higher scale of observation. Considering the shape of the surface and the directions of the effective plastic flow vectors, the effective failure surface can be divided into 6 parts with smoothly pronounced curvature, connected by more sharply curved transition regions. These 6 surface parts are indicated in Figure 6 by dashed curves with circled numbers, and based thereon selected stress points (red circles), for which related failure modes are shown in Figure 7. To each surface part, a certain failure mechanism with a 


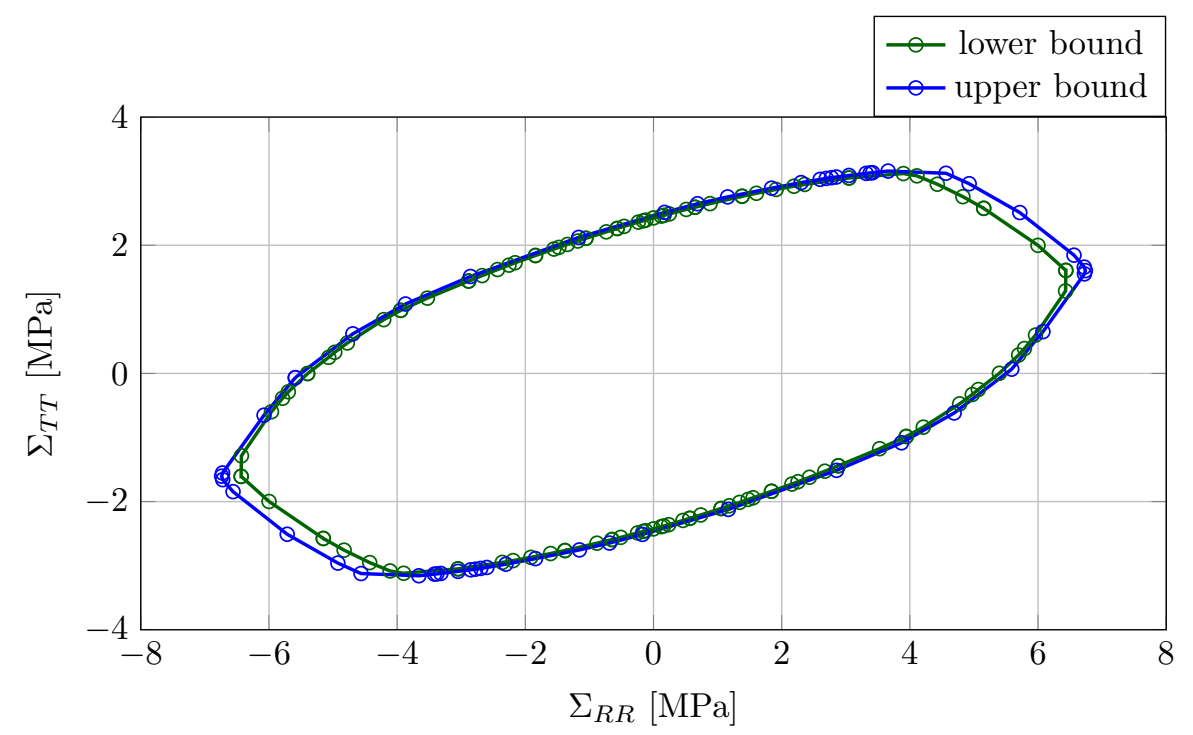

(a)

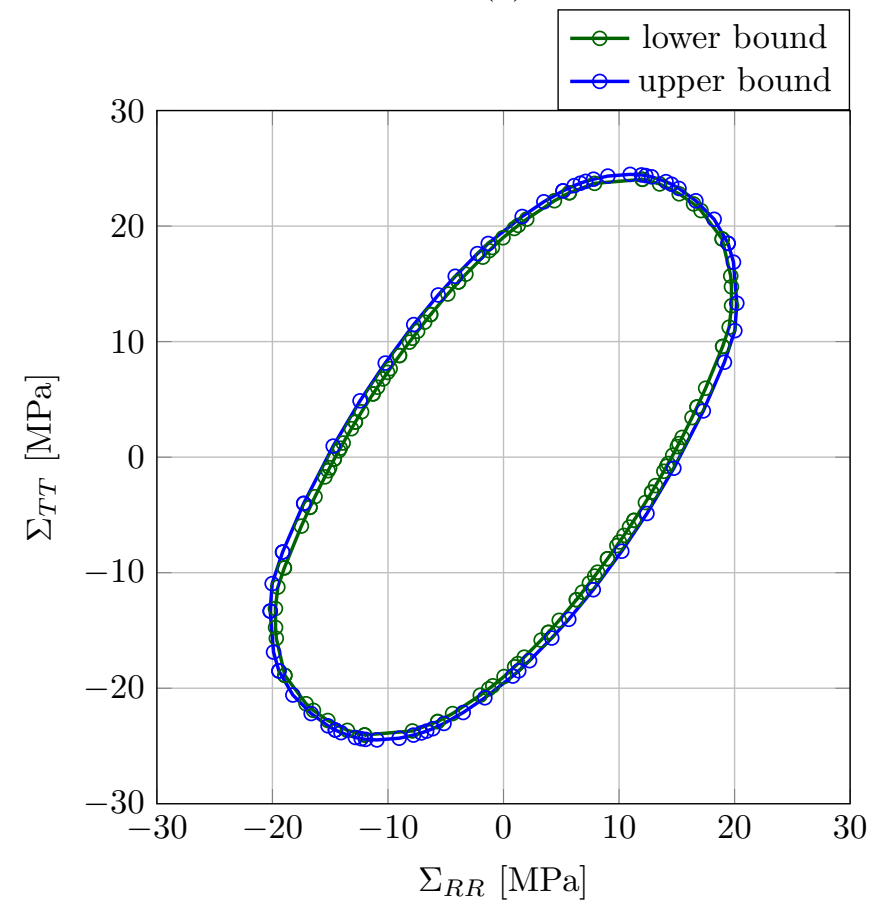

(b)

Figure 5: Lower and upper bound effective failure surfaces of (a) the earlywood unit cell and (b) the latewood unit cell.

distinct failure mode can be assigned. Noting that there are qualitatively similar mechanisms in opposite tension and compression regions, the number of strongly differing mechanisms reduces to 3. The identification of failure mechanisms and failure modes will be discussed in the following (the referenced failure mechanisms can be found in Figure 7): 


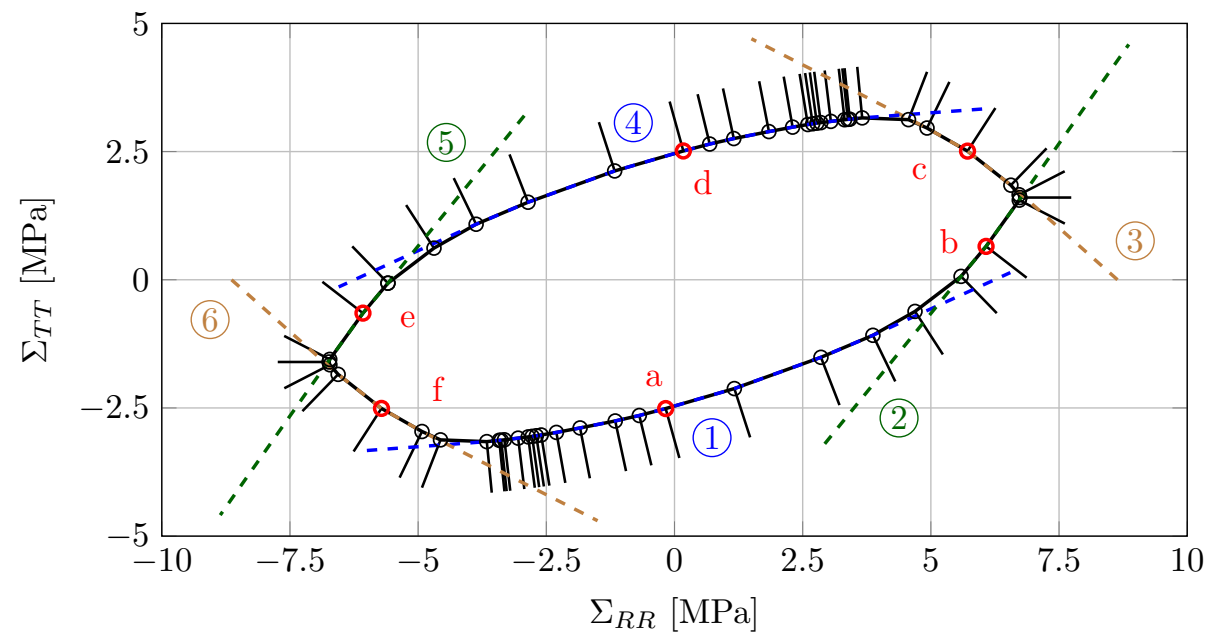

Figure 6: The effective upper bound failure surface for the earlywood unit cell, including velocity vectors, pointing in the direction of the related effective plastic strain rate, and dashed curves dividing the failure surface into 6 parts.

1. Failure mechanisms associated with surface parts (1) and (4) are mainly affected by loading in the $T$-direction, as can be clearly seen in mechanisms (a) and (d), respectively. Failure is characterised by two plastic hinges in each of the four wooden cell webs running in the $R$-direction. Plastification takes place in the middle lamella as well as the cell wall layers. In addition, the middle lamella region in between these two hinges is also plastified, allowing the occuring kinematics.

2. In contrast, failure mechanisms (b) and (e) associated with surface parts (2) and (5) are mainly characterised by the loading in the $R$-direction. Strongly delineated failure regions are initiated in the cell wall layer, and then, penetrating through the middle lamella layer in the $T$-direction. The majority of energy is dissipated in the stronger cell wall material, leading to the significantly higher effective strength in the $R$-direction.

3. The biaxial character of the loading can be identified in failure mechanisms (c) and (f), associated with surface parts (3) and (6). The main plastification occurs in the middle lamella, including small shear failure surfaces running in $R$-direction, accompanied by locally demarcated failure through the cell wall layers.

\section{Latewood}

In Figure 8 the effective upper bound failure surface of the latewood unit cell is plotted once again, also including the directions of the effective plastic strain rates. Similar to the results from the earlywood unit cell, the effective plastic flow rate of the latewood unit cell reflects the underlying associated flow rule used in the limit analysis. Again, the failure surface can be divided into 6 parts with different characteristics. The corresponding 3 independent failure mechanisms are described in the following (the referenced failure mechanisms can be found in Figure 9):

1. Failure mechanisms (a) and (d) associated with surface parts (1) and (4) are characterised by thin shear bands running diagonally through the middle lamella together with plastic hinges 
in the cell wall layer. Macroscopic cracks can be expected to run diagonally through the latewood cell structure, as indicated by the shear bands.

2. The similar looking mechanisms (b) and (e) are linked to surface parts (2) and (5). The difference, compared with other failure mechanisms, becomes obvious when taking directions of effective/macroscopic plastic strain rates into account, which align almost horizontally. Thus, failure in the $R$-direction through the latewood can be expected, including distinct initiation of failure regions in the cell wall layer.

3. The third clearly different mode, failure mechanisms (c) and (f), is associated with surface parts (3) and (6). Plastification is dominated by shear failure regions, which extend in a vertical direction through the entire cell wall.

\subsection{Application of limit analysis at clear wood scale}

The next higher observation scale, the clear wood scale (see Figure 1), also exhibits a repetitive structure, consisting of homogeneous earlywood and latewood layers. Thus, this structure can also be represented by a unit cell with appropriate periodic boundary conditions. At this observation scale, we are considering the $R L$-plane, as illustrated in Figure 1, because experimental data from biaxial tests are available in this plane in Eberhardsteiner (1995). The unit cell at this scale is defined as a layered laminated structure with earlywood and latewood layers aligning in the same orientation, as shown in Figure 10. Following the above-mentioned experiments, five different principal material orientations with respect to principal effective stresses $\Sigma_{1}$ and $\Sigma_{2}$, defined by the angle $\alpha$, are investigated numerically.

It has been discussed by Eberhardsteiner (1995), and Mackenzie-Helnwein et al. (2003) that the material behaviour of clear wood can be approximated by the orthotropic Tsai-Wu failure criterion. Therefore, this criterion is assigned to both the earlywood and latewood layers. Following the idea of upscaling, strength parameters should be determined by results from the previous scale of observation, the annual ring scale.

In this paper, the $L R$-plane is considered at the clear wood scale, while the $R T$-plane is considered at the annual ring scale. As a result, at the current scale, only effective strengths in the $R$ direction can be used from the limit analysis simulations at the previous scale (Section 3.1). For earlywood and latewood strengths in the $L$-direction, experimentally obtained values in Sinn et al. (2001); Gindl and Teischinger (2002) are employed.

Accordingly the following Tsai-Wu strength parameters were assigned to the earlywood layer:

$$
\begin{aligned}
F_{1} & =-0.0116 \mathrm{MPa}^{-1} & F_{2} & =0 \mathrm{MPa}^{-1} \\
P_{11} & =5.0 \mathrm{E}-4 \mathrm{MPa}^{-2} & P_{22} & =3.38 \mathrm{E}-2 \mathrm{MPa}^{-2} \\
P_{12} & =0 \mathrm{MPa}^{-2} & &
\end{aligned}
$$

and to the latewood layer:

$$
\begin{aligned}
F_{1} & =-0.0093 \mathrm{MPa}^{-1} & F_{2} & =0 \mathrm{MPa}^{-1} \\
P_{11} & =1.0 \mathrm{E}-4 \mathrm{MPa}^{-2} & P_{22} & =4.70 \mathrm{E}-3 \mathrm{MPa}^{-2} \\
P_{12} & =0 \mathrm{MPa}^{-2} & &
\end{aligned}
$$


With respect to the shear strength in the $L R$-plane, no experimental data, separately for the earlywood and latewood, could be found. Thus, two different approaches were used to estimate suitable values. First, simply the shear strength of clear wood, given in Gindl and Teischinger (2002), is assigned to both layers. Second, the clear wood shear strength is linearly scaled based on the considered densities of the earlywood and latewood materials. The so-obtained Tsai-Wu parameters are listed below:

First approach:

$P_{66}=1.35 \mathrm{E}-2 \mathrm{MPa}^{-2}$ for both earlywood and latewood layers

Second approach:

$P_{66}=2.44 \mathrm{E}-2 \mathrm{MPa}^{-2}$ for earlywood layer

$P_{66}=3.70 \mathrm{E}-3 \mathrm{MPa}^{-2}$ for latewood layer

\subsubsection{Determination of effective failure surfaces}

The lower bound formulation, Eq. (21), and the dual upper bound formulation, Eq. (43), are now applied to the unit cell models defined in Figure 10, delivering effective failure stresses and failure mechanisms under various loading situations. Failure modes obtained from the upper bound simulations under 3 different loading conditions, are shown in Figure 11 to illustrate typical patterns of failure regions and the plastic flow mechanism. From the corresponding deformation fields, periodic velocity boundary conditions can be identified very well. However, at this scale of observation, failure modes are not the main focus and will not be discussed in detail.

Subsequently, similar to the previous scale, various loading situations are applied to the clear wood unit cell. The number of simulations and the corresponding (very low) computing times are given in Table 1.

\begin{tabular}{|c||c|c|c|c|c|}
\hline unit cell & 1 & 2 & 3 & 4 & 5 \\
\hline number of elements & 450 & 806 & 622 & 582 & 490 \\
\hline number of load situations/simulations & 860 & 660 & 852 & 860 & 860 \\
\hline total computing time [min] & 57.7 & 54.1 & 74.7 & 73.1 & 67.6 \\
\hline
\end{tabular}

Table 1: Simulation program at the clear wood scale.

Finally, the obtained effective failure surfaces for the five different annual ring orientations are compared to the experimentally obtained failure stress states for clear wood that were presented in Eberhardsteiner (1995). The result is plotted in Figure 12. The numerical limit analysis results agree very well with the experimental results, qualitatively as well as quantitatively. The numerical results tend to overestimate the experimental ones, which is in perfect accordance with the fact that limit analysis is based on an ideal perfectly plastic response of the material, rather than a brittle one. Nevertheless, the results provide meaningful predictions of the clear wood strength, also showing that wood must have some ductile potential, even under tension-dominated loading. 


\section{Summary and conclusions}

This work shows the first time application of numerical limit analysis formulations to microstructures of wood. With respect to the three objectives specified in Section 1, the main findings can be summarized as follows:

- The orthotropic Tsai-Wu failure criterion was formulated as a second-order cone constraint, and thus, easily incorporated into numerical limit analysis formulations that use cone programming. To the author's knowledge, this failure criterion has been combined for the first time with periodic boundary conditions, as anti-periodic traction fields and periodic velocity fields. Both were successfully enforced in lower bound and upper bound formulations, respectively.

- The newly formulated limit analysis formulations were successfully applied to unit cells at the annual ring scale and the clear wood scale of wood. Through variation of loading conditions, effective failure surfaces in the macroscopic stress space were obtained very stably and efficiently at both observation scales. For all simulations the small difference between the lower and upper bound results (the bracketing gap) was highly satisfactory.

- The comparison between numerically-obtained effective failure surfaces at the clear wood scale and experimental results from biaxial testing of Norway spruce clear wood showed very good agreement, confirming the proposed method as very promising.

Based on these results it can be concluded, that due to its efficiency and simplicity and good prediction quality, the presented limit analysis concept should be able to play an important role in the strength prediction of wood and wood-based products. Future work is being devoted to the extension of the presented approach to the third dimension and the application to next higher scales of observation, such as the scales of the wooden board and the wood-based product Hochreiner et al. $(2013,2014)$. With efficient and stable strength estimates of wooden boards, also stochastic considerations as proposed in Kandler et al. (2015); Füssl et al. (2016) for elasticity, can be tackled for the effective strength properties of wood-based products. Even if it is not used to directly access the load bearing capacity of wood, it seems to be a very well suited method to provide a first information on failure mechanisms, e.g., as input to brittle failure simulations, which would finally provide a conservative design strength for a certain knot group, as it is done in Jenkel and Kaliske (2014) for example. Therein, plastic finite element calculations are performed (which could be replaced by more efficient limit analysis calculations) to gain information about failure zones around/between knots and this information is used to define the location of cohesive elements, subsequently delivering brittle strength estimates. Moreover, to get an initial strength evaluation of a defect or for the identification of critical defects, limit analysis simulations would be sufficient. In parallel, results of the proposed limit analysis concept have just been compared to results from extended finite element simulations as well as to elastic limit states obtained in the framework of continuum micromechanics in Füssl et al. (2017), showing a very promising performance of limit analysis with respect to the prediction of failure mechanisms and high time efficiency. 


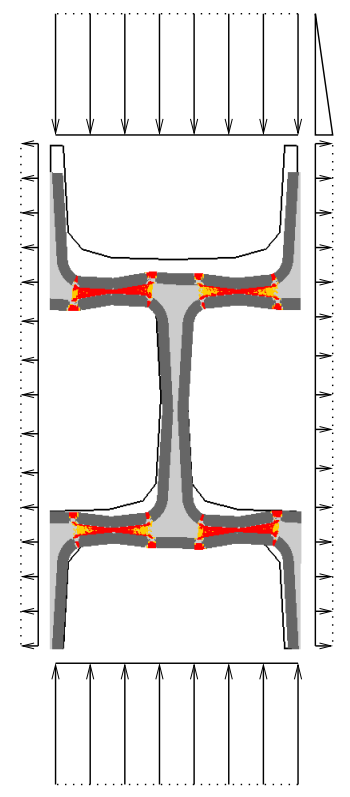

(a) $\dot{V}_{R}: \dot{V}_{L}=1:-7$

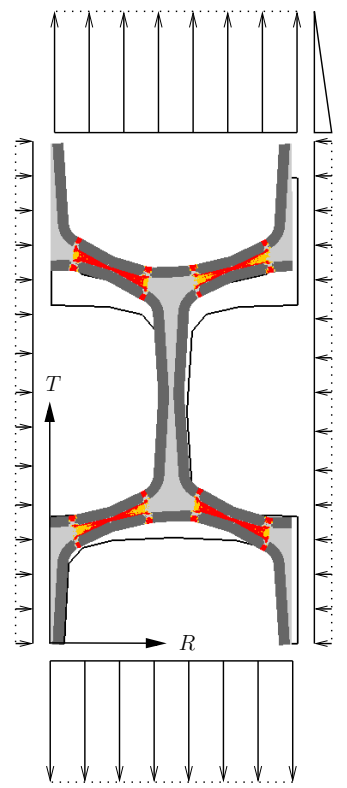

(d) $\dot{V}_{R}: \dot{V}_{L}=-1: 7$
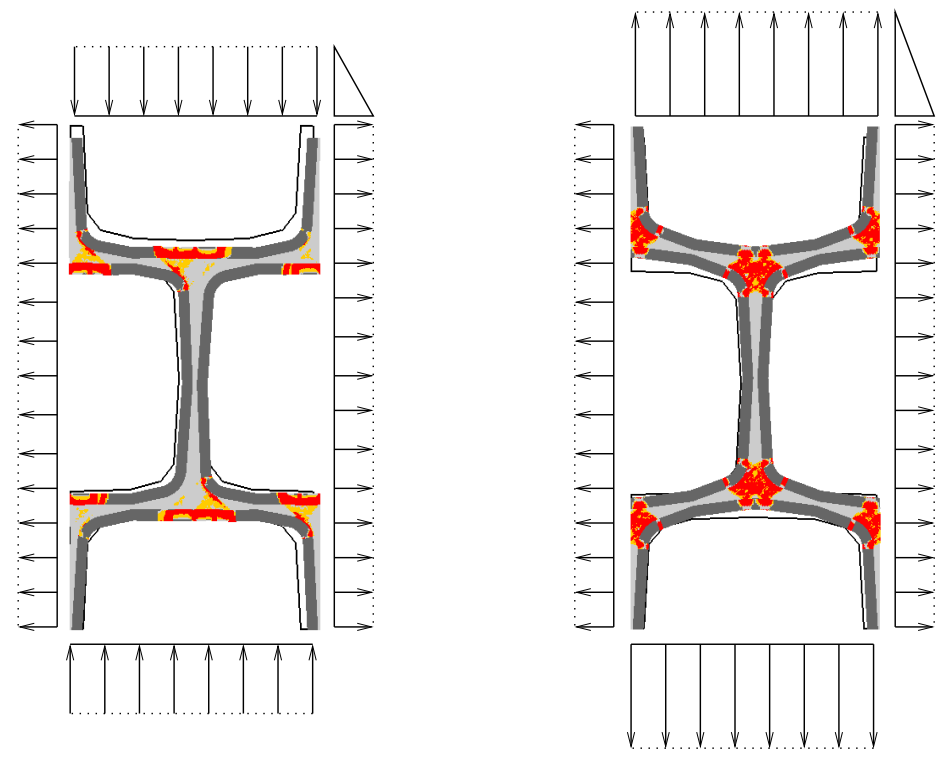

(b) $\dot{V}_{R}: \dot{V}_{L}=1:-2$

(c) $\dot{V}_{R}: \dot{V}_{L}=1: 3$
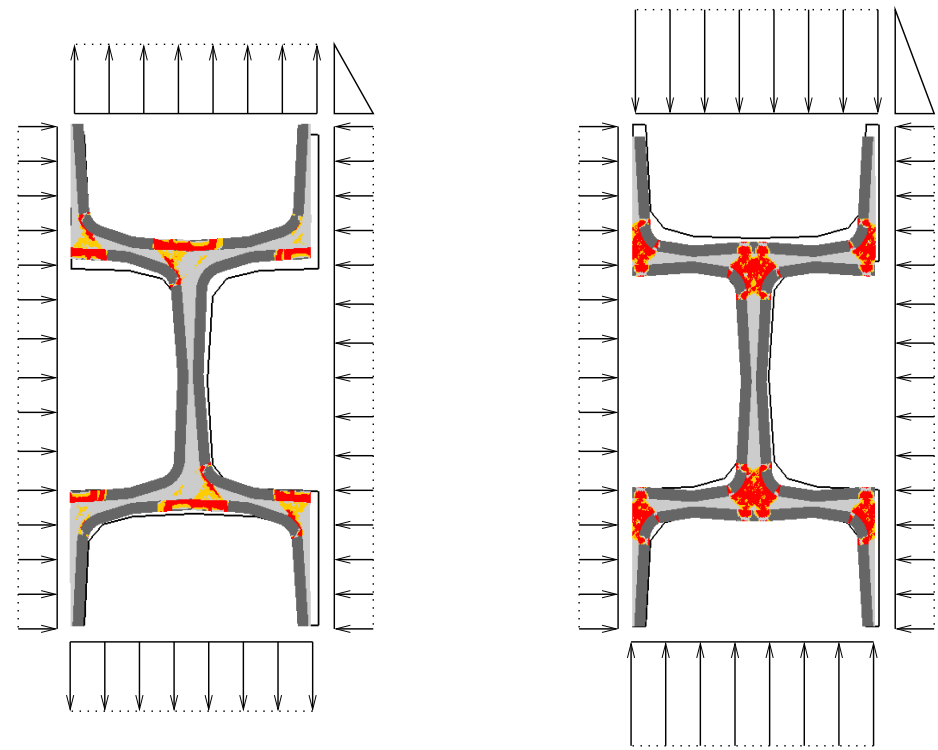

(f) $\dot{V}_{R}: \dot{V}_{L}=-1:-3$

Figure 7: Selected failure mechanisms of the earlywood unit cell obtained from the upper bound analysis. Failure modes corresponding to effective stress states marked with red circles in Figure 6. Anti-periodic velocity conditions are applied and the velocity field is not constant over each boundary surface indicated as dash line. The loading condition is prescribed as the ratio between $\dot{V}_{R}$ and $\dot{V}_{L}$, denoting the area-averaged velocities in $R$ and $T$ direction over the corresponding boundary surfaces, which are equivalent to the macroscopic strain rate $\dot{E}_{R R}$ and $\dot{E}_{L L}$ (in Eq. (30)), respectively. 


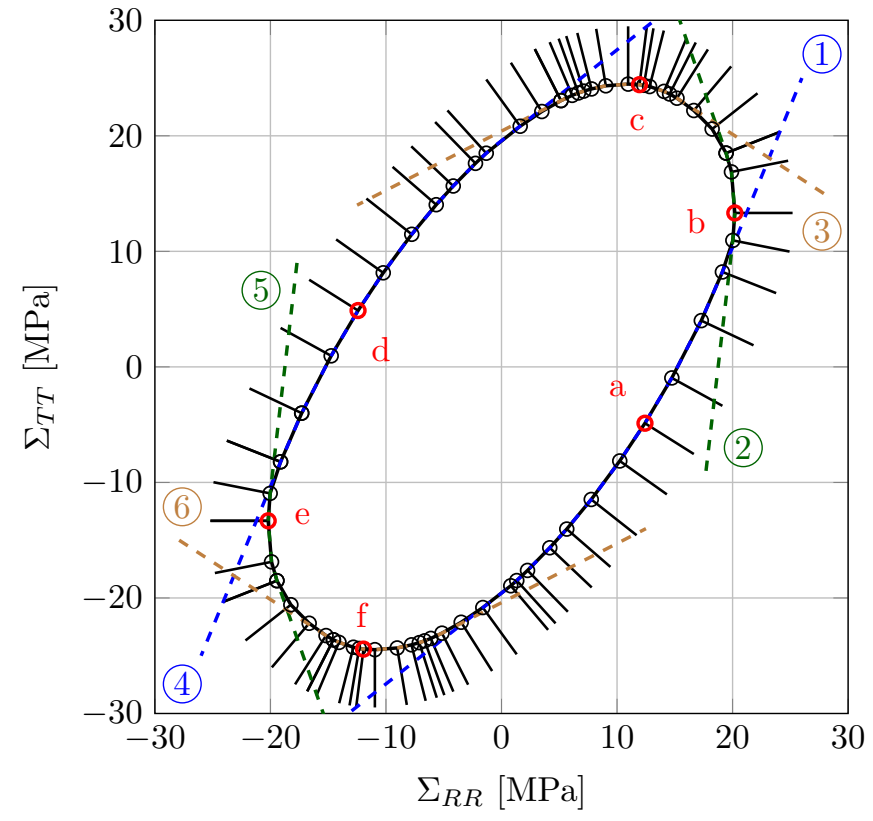

Figure 8: The effective upper bound failure surface for the latewood unit cell, including black lines at every stress point, pointing into the direction of the related effective plastic strain rate. 


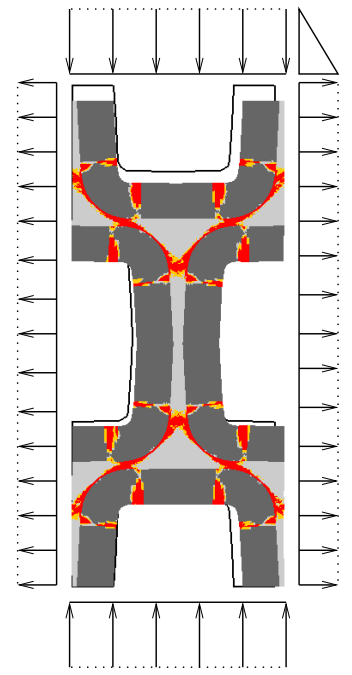

(a) $\dot{V}_{R}: \dot{V}_{L}=1:-1.6$

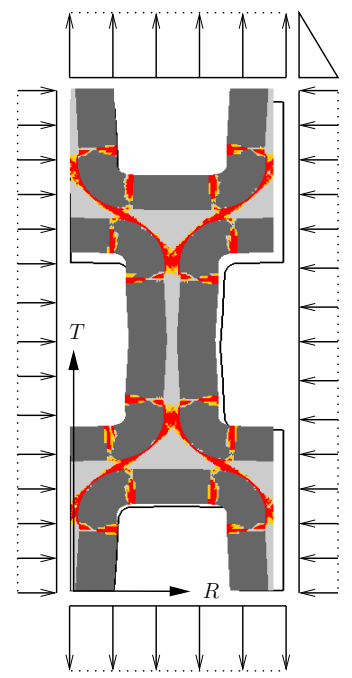

(d) $\dot{V}_{R}: \dot{V}_{L}=-1: 1.6$

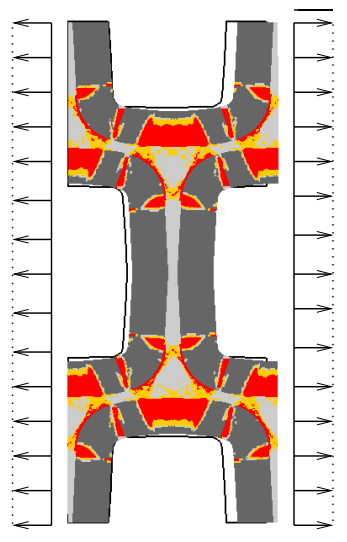

(b) $\dot{V}_{R}: \dot{V}_{L}=1: 0$

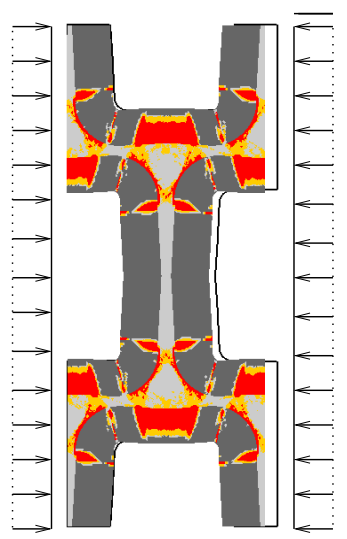

(e) $\dot{V}_{R}: \dot{V}_{L}=-1: 0$

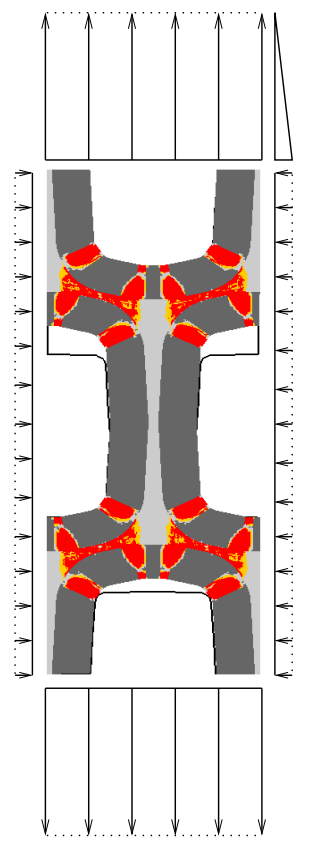

(c) $\dot{V}_{R}: \dot{V}_{L}=-1: 10$

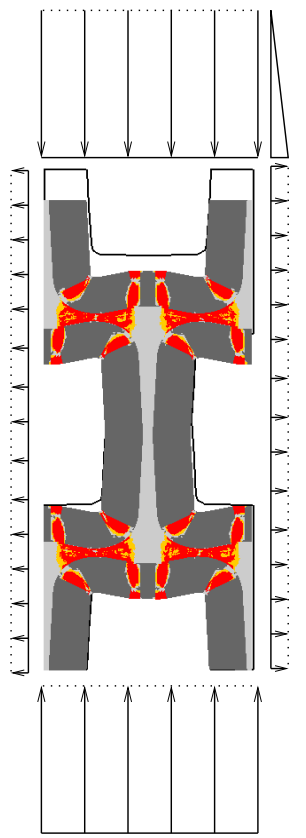

(f) $\dot{V}_{R}: \dot{V}_{L}=1:-10$

Figure 9: Selected failure mechanisms of the latewood unit cell obtained from upper bound simulations. Failure modes belonging to the effective stress states marked with red circles in Figure 8. Anti-periodic velocity conditions are applied and the velocity field is not constant over each boundary surface indicated as dash line. The loading condition is prescribed as the ratio between $\dot{V}_{R}$ and $\dot{V}_{L}$, denoting the area-averaged velocities in $R$ and $T$ direction over the corresponding boundary surfaces, which are equivalent to the macroscopic strain rate $\dot{E}_{R R}$ and $\dot{E}_{L L}$ (in Eq. (30)), respectively. 


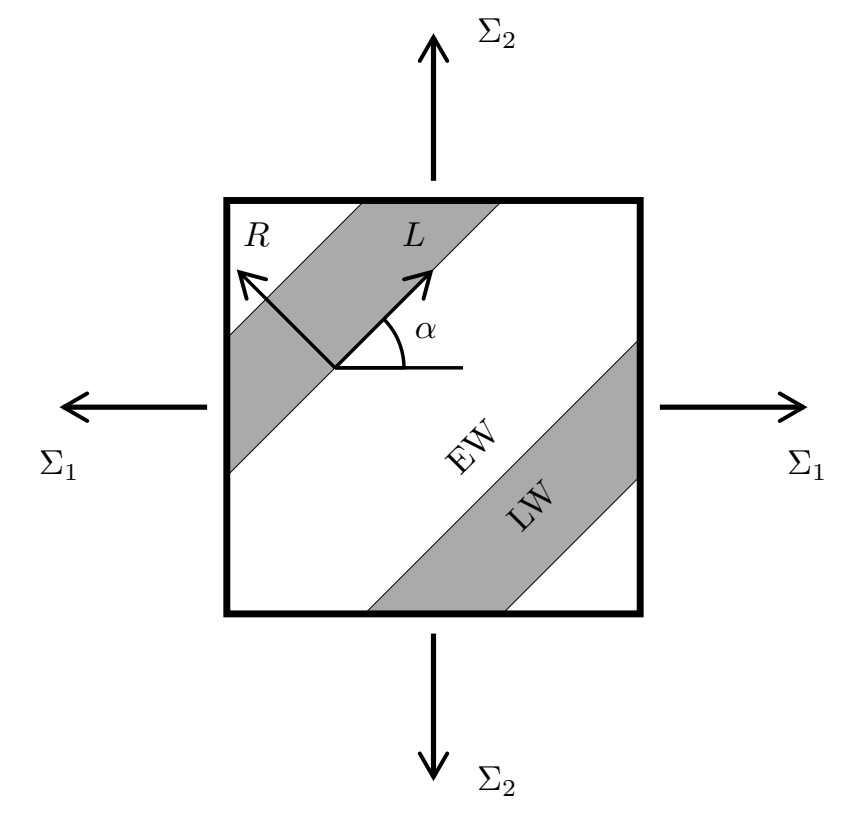

\begin{tabular}{|c||c|c|c|c|c|}
\hline unit cell & 1 & 2 & 3 & 4 & 5 \\
\hline$\alpha\left[^{\circ}\right]$ & 0 & 7.5 & 15 & 30 & 45 \\
\hline
\end{tabular}

Figure 10: Representative unit cell for the clear wood scale, consisting of homogeneous earlywood and latewood layers. Five different annual ring orientations, defined by the angle $\alpha$ have been investigated.

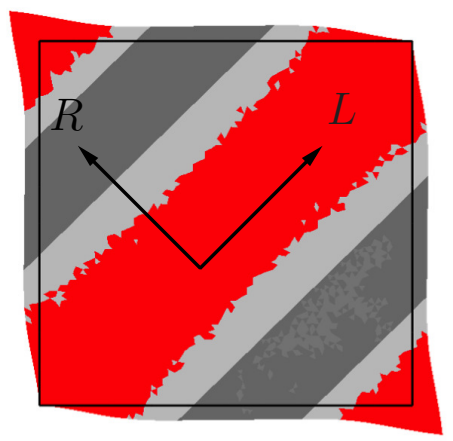

(a)

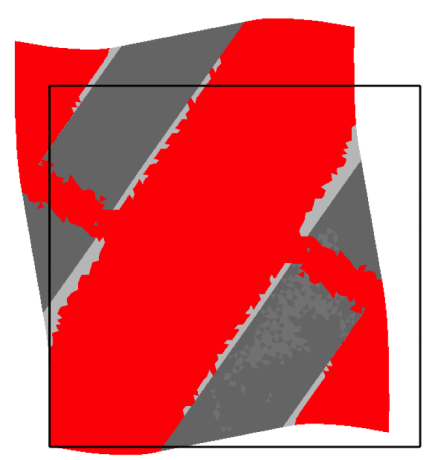

(b)

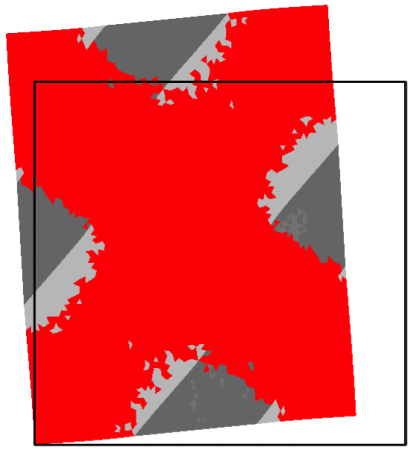

(c)

Figure 11: Illustrative failure mechanisms of the clear wood unit cell obtained from upper bound simulations for 3 different effective loading ratios $\dot{E}_{1} / \dot{E}_{2}$ : (a) 1.0, (b) -0.8, and (c) -1.0. 


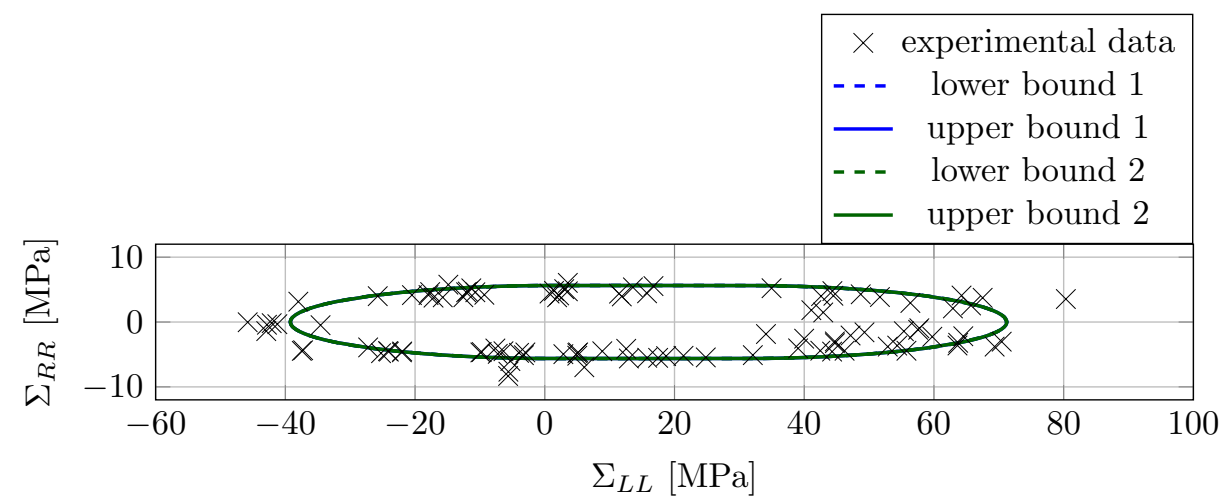

(a) $\alpha=0^{\circ}$

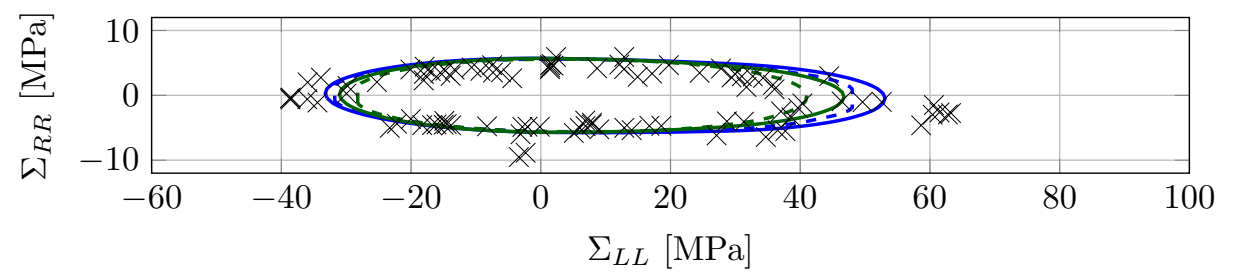

(b) $\alpha=7.5^{\circ}$

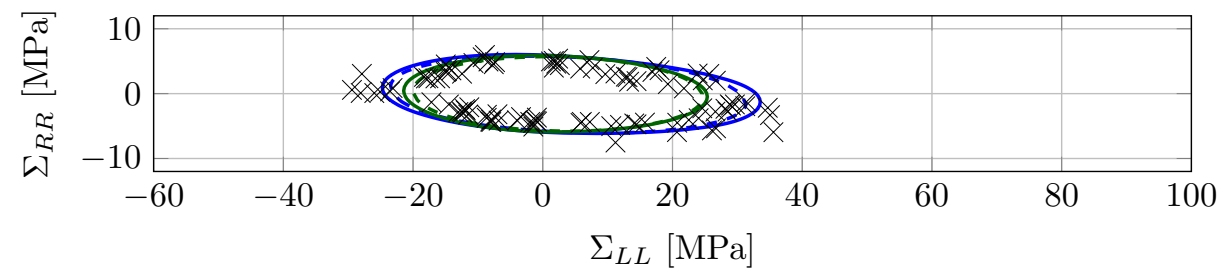

(c) $\alpha=15^{\circ}$

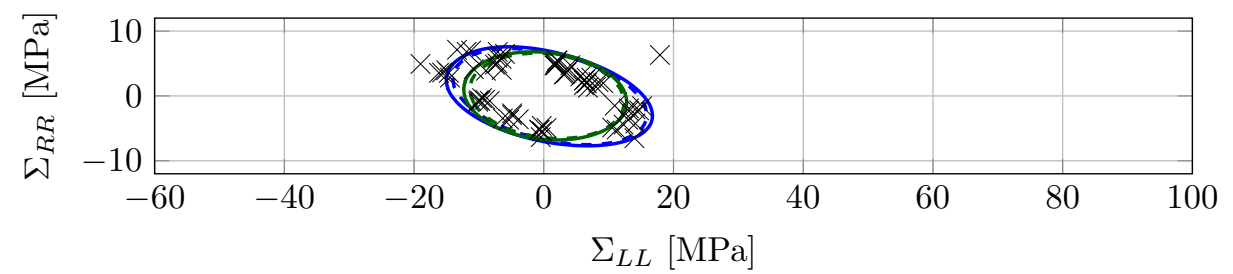

(d) $\alpha=30^{\circ}$

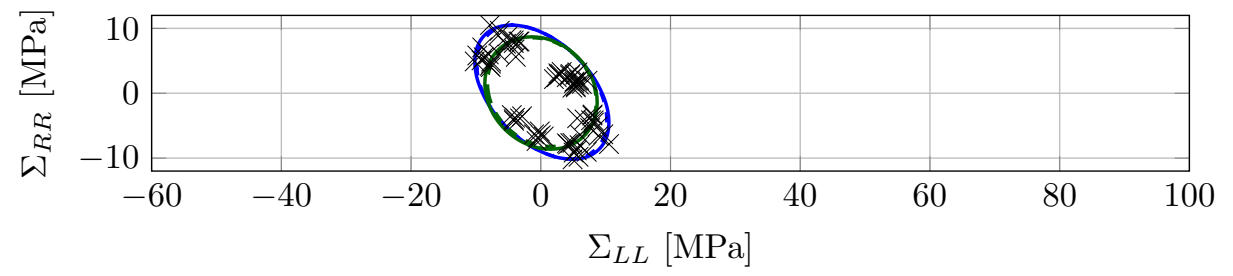

(e) $\alpha=45^{\circ}$

Figure 12: Comparison of numerically-obtained lower and upper bound effective failure surfaces of clear wood with experimentally-obtained failure stress states in Eberhardsteiner (1995) for Norway spruce clear wood. Blue curves are obtained with the first approach and green curves with the second approach for the choice of shear strengths (see Eq. (48)). 


\section{Appendix A. Second-order cone programming}

The definition of the cone set $\mathscr{C}$ is that,

$$
\forall \mathbf{x} \in \mathscr{C} \text { and } \lambda \geq 0 \Longleftrightarrow \lambda \mathbf{x} \in \mathscr{C}
$$

and then its dual cone $\mathscr{C}^{*}$ can be defined as,

$$
\mathbf{x}^{\top} \mathbf{y} \geq 0, \forall \mathbf{x} \in \mathscr{C} \Longleftrightarrow \mathbf{y} \in \mathscr{C}^{*}
$$

where $\mathbf{x} \in \mathfrak{R}^{n}$ and $\mathbf{y} \in \mathfrak{R}^{n}$. If $\mathscr{C}=\mathscr{C}^{*}$ holds, the cone is considered as self-dual.

The most common second-order cones is the quadratic cone $\mathscr{C}$,

$$
\mathscr{C}=\left\{\mathbf{x}: x_{1} \geq\left\|\mathbf{x}_{2: d}\right\|, x_{1} \geq 0\right\}
$$

and the rotated quadratic cone,

$$
\mathscr{C}=\left\{\mathbf{x}: 2 x_{1} x_{2} \geq\left\|\mathbf{x}_{3: d}\right\|, x_{1} \geq 0, x_{2} \geq 0\right\}
$$

where $\mathbf{x}_{m: n}=\left[x_{m} \ldots x_{n}\right]^{\top} \in \mathfrak{R}^{n-m}$, and both the two second-order cones are self-dual.

Then the optimization problem of the second-order cone programming (SOCP) can be expressed as

$$
\begin{array}{ll}
\min & \mathbf{c}^{\top} \mathbf{x} \\
\text { s.t. } & \mathbf{A x}=\mathbf{b} \\
& \mathbf{x} \in \mathscr{C}
\end{array}
$$

where $\mathbf{b} \in \mathfrak{R}^{m}, \mathbf{c} \in \mathfrak{R}^{n}, \mathbf{A} \in \mathfrak{R}^{m \times n}$ and $\mathscr{C}$ is the second-order cone.

The dual optimization problem is

$$
\begin{array}{ll}
\max & \mathbf{b}^{\top} \mathbf{z} \\
\text { s.t. } & \mathbf{A}^{\top} \mathbf{z}+\mathbf{y}=\mathbf{c} \\
& \mathbf{y} \in \mathscr{C}^{*} .
\end{array}
$$

where $\mathbf{z} \in \mathfrak{R}^{m}$ and $\mathscr{C}^{*}$ is the dual cone of $\mathscr{C}$.

$\mathrm{SOCP}$ can be regarded as a generalization of classical linear programming where the linear constraints are supplemented by specialised nonlinear constraints in the form of the cone constraints. The resulting optimization problem can be solved efficiently with primal-dual algorithms based on the interior-point method.

\section{Appendix B. Matrix representation of the limit analysis formulation}

To solve the optimization problem, it is very convenient and efficient to use the commercial SOCP solver MOSEK. Therefore, the optimization formulation has to be reformulated in the matrix form suitable for input to MOSEK. 
For the lower bound problem, the SOCP formulation in Eq. (21) is transformed into

$\max \beta$

$$
\begin{aligned}
& L E \times 9+L E \times 9+L E \times 15+1 \\
& \begin{array}{l}
L E \times 2 \\
L D \times 4 \\
L P \times 2 \\
1 \\
1 \\
L E \times 9 \\
L E \times 15
\end{array}\left(\begin{array}{ccccccc}
\mathbf{A}_{L B}^{e q} & \vdots & \mathbf{0} & \vdots & \mathbf{0} & \vdots & \mathbf{0} \\
\mathbf{A}_{L B}^{d i s} & \vdots & \mathbf{0} & \vdots & \mathbf{0} & \vdots & \mathbf{0} \\
\mathbf{A}_{L B}^{p e r} & \vdots & \mathbf{0} & \vdots & \mathbf{0} & \vdots & \mathbf{0} \\
\mathbf{b}_{x}^{p e r \top} & \vdots & \mathbf{0} & \vdots & \mathbf{0} & \vdots & -f_{t, x} \\
\mathbf{b}_{y}^{p e r \top} & \vdots & \mathbf{0} & \vdots & \mathbf{0} & \vdots & -f_{t, y} \\
-\mathbf{R} & \vdots & \mathbf{I} & \vdots & \mathbf{0} & \vdots & \mathbf{0} \\
\mathbf{0} & \vdots & -\mathbf{B}^{s o c} & \vdots & \mathbf{I} & \vdots & \mathbf{0}
\end{array}\right)\left(\begin{array}{c}
\mathbf{q}_{\sigma} \\
\mathbf{q}_{\sigma}^{\prime} \\
\mathbf{s}^{s o c} \\
\beta
\end{array}\right)=\left(\begin{array}{c}
\mathbf{0} \\
\mathbf{0} \\
\mathbf{0} \\
\mathbf{0} \\
\mathbf{0} \\
\mathbf{0} \\
\mathbf{a}^{s o c}
\end{array}\right)
\end{aligned}
$$

in which the objective function is the load multiplier $\beta$.

Similarly, the upper bound optimization can be derived as the primal form,

$\min W_{\text {int }}$

$$
\begin{aligned}
& \begin{array}{c}
U N \times 2 \\
U E \times 9 \\
U S \times U C \\
U S \times U C \\
U P \times 2
\end{array}\left(\begin{array}{ccccc}
\mathbf{A}_{U B}^{e q}{ }^{\top} & \vdots & \mathbf{R}_{\sigma}^{\top} & \vdots & \mathbf{0} \\
\mathbf{0} & \vdots & \mathbf{0} & \vdots & -\mathbf{I} \\
\mathbf{0} & \vdots & -\mathbf{I} & \vdots & \mathbf{B}^{s o c \top} \\
-\mathbf{A}_{U B}^{p e r} & \vdots & \mathbf{0} & \vdots & \mathbf{0}
\end{array}\right)\left(\begin{array}{c}
\mathbf{q}_{\dot{u}} \\
-\mathbf{q}_{\dot{\varepsilon}}^{\prime} \\
\mathbf{e}^{s o c}
\end{array}\right)=\left(\begin{array}{c}
\mathbf{0} \\
-\mathbf{e}^{s o c} \\
\mathbf{0} \\
\dot{\mathbf{E}}_{U B} \mathbf{l}_{U B}
\end{array}\right) \\
& \text { s.t. }
\end{aligned}
$$

and the dual form,

$$
\begin{aligned}
& \max W_{\text {ext }}
\end{aligned}
$$

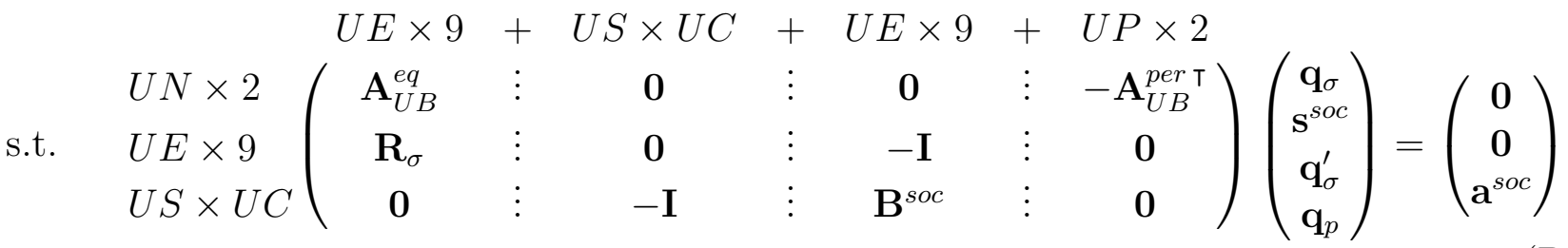

from Eq. (41) and Eq. (43) respectively. The duality is clear comparing to the definitions in Eq. (A.5) and Eq. (A.6).

\section{Acknowledgments}

Financial support for this work in the framework of the PhDSchool DokInHolz funded by the Austrian Federal Ministry of Science, Research and Economy and the Austrian Association of Wood Industries is gratefully acknowledged. We also gratefully acknowledge the financial support of this work by the Austrian Science Fund (FWF) through the Erwin Schrödinger Fellowship J3748-N30. 
Aicher, S., Gustafsson, P. J., Haller, P., Petersson, H., 2002. Fracture mechanics models for strength analysis of timber beams with a hole or a notch - a report of RILEM TC-133. Tech. rep., Structural Mechanics.

Anderheggen, E., Knöpfel, H., 1972. Finite element limit analysis using linear programming. International Journal of Solids and Structures 8 (12), 1413 - 1431.

Andersen, E., Roos, C., Terlaky, T., 2003. On implementing a primal-dual interior-point method for conic quadratic optimization. Mathematical Programming 95 (2), 249-277.

Bader, T. K., Hofstetter, K., Hellmich, C., Eberhardsteiner, J., 2010. Poromechanical scale transitions of failure stresses in wood: from the lignin to the spruce level. Journal of Applied Mathematics and Mechanics 90 (10-11), 750-767.

Bader, T. K., Hofstetter, K., Hellmich, C., Eberhardsteiner, J., 2011. The poroelastic role of water in cell walls of the hierarchical composite softwood. Acta mechanica 217 (1-2), 75-100.

Ciria, H., Peraire, J., Bonet, J., 2008. Mesh adaptive computation of upper and lower bounds in limit analysis. International Journal for Numerical Methods in Engineering 75 (8), 899-944.

Danielsson, H., Gustafsson, P. J., 2014. Fracture analysis of glued laminated timber beams with a hole using a 3D cohesive zone model. Engineering Fracture Mechanics 124-125, 182 - 195.

Denton, S. R., Morley, C. T., 2000. Limit analysis and strain-softening structures. International Journal of Mechanical Sciences 42 (3), $503-522$.

Drucker, D. C., Greenberg, H. J., Prager, W., 1951. The safety factor of an elastic-plastic body in plane strain. Journal of Applied Mechanics 18, 371-378.

Drucker, D. C., Prager, W., Greenberg, H. J., 1952. Extended limit design theorems for continuous media. Quarterly of Applied Mathematics 9, 381-389.

Eberhardsteiner, J., 1995. Biaxial testing of orthotropic materials using electronic speckle pattern interferometry. Measurement 16 (3), 139 - 148.

Fengel, D., Wegener, G., 1983. WOOD Chemistry, Ultrastructure, Reactions. Verlag Kessel.

Füssl, J., Kandler, G., Eberhardsteiner, J., 2016. Application of stochastic finite element approaches to wood-based products. Archive of Applied Mechanics 86 (1-2), 89-110.

Füssl, J., Lackner, R., Eberhardsteiner, J., Mang, H., 2008. Failure modes and effective strength of two-phase materials determined by means of numerical limit analysis. Acta Mechanica 1-4 (195), $185-202$.

Füssl, J., Li, M., Lukacevic, M., Eberhardsteiner, J., Martin, C. M., 2017. Comparison of unit cell-based computational methods for perdicting the strength of wood. Engineering structures $141,427-443$. 
Ghosh, S., Lee, K., Raghavan, P., 2001. A multi-level computational model for multi-scale damage analysis in composite and porous materials. International Journal of Solids and Structures $38(14), 2335-2385$.

URL http://www.sciencedirect.com/science/article/pii/S0020768300001670

Gindl, W., Gupta, H., Lichtenegger, T. S. H., Fratzl, P., 2004. Mechanical properties of spruce wood cell walls by nanoindentation. Applied Physics A: Materials Science \& Processing 79, 2069-2073.

Gindl, W., Teischinger, A., 2002. Axial compression strength of norway spruce related to structural variability and lignin content. Composites Part A: Applied Science and Manufacturing 33 (12), $1623-1628$.

Gloimüller, S., Borst, K., Bader, T., Eberhardsteiner, J., 2012. Determination of the linear elastic stiffness and hygroexpansion of softwood by a multilayered unit cell using porommechanics. Interaction and Multiscale Mechanics 5 (3), 229-265.

Guindos, P., 2011. Three-dimensional finite element models to simulate the behavior of wood with presence of knots, appliying the flow-grain analogy and validation with close range photogrammetry. Ph.D. thesis, University of Santiago de Compostela, Department of Agroforestry Engineering.

Hautefeuille, M., Colliat, J.-B., Ibrahimbegovic, A., Matthies, H., Villon, P., 2012. A multi-scale approach to model localized failure with softening. Computers \& Structures 9495, 83 - 95.

URL https://www.sciencedirect.com/science/article/pii/S0045794911002926

Hill, R., 1951. On the state of stress in a plastic-rigid body at the yield point. Philosophical Magazine 42, 868-875.

Hochreiner, G., Füssl, J., Eberhardsteiner, J., 2013. Cross-laminated timber plates subjected to concentrated loading - experimental identification of failure mechanisms. Strain (50), 68-81.

Hochreiner, G., Füssl, J., Serrano, E., Eberhardsteiner, J., 2014. Influence of wooden board strength class on the performance of cross-laminated timber plates investigated by means of full-field deformation measurements. Strain (50), 161-173.

Hofstetter, K., Hellmich, C., Eberhardsteiner, J., 2005. Development and experimental validation of a continuum micromechanics model for the elasticity of wood. European Journal of Mechanics - A/Solids 24 (6), 1030 - 1053.

Hofstetter, K., Hellmich, C., Eberhardsteiner, J., 2007. Micromechanical modeling of solid-type and plate-type deformation patterns within softwood materials. a review and an improved approach. Holzforschung 61 (4), 343-351.

Hofstetter, K., Hellmich, C., Eberhardsteiner, J., Mang, H. A., 2008. Micromechanical estimates for elastic limit states in wood materials, revealing nanostructural failure mechanisms. Mechanics of Advanced Materials and Structures 15 (6-7), 474-484. 
Jenkel, C., Kaliske, M., 2014. Finite element analysis of timber containing branches - an approach to model the grain course and the influence on the structural behaviour. Engineering Structures $75,237-247$.

Kandler, G., Füssl, J., Eberhardsteiner, J., 2015. Stochastic finite element approaches for woodbased products: theoretical framework and review of methods. Wood Science and Technology 49 (5), 1055-1097.

Kollmann, F., 1951. Technologie des Holzes und der Holzwerkstoffe. Springer-Verlag Berlin Heidelberg.

Krabbenhøft, K., Damkilde, L., 2000. Limit Analysis Based On Lower-Bound Solutions And Nonlinear Yield Criteria. Civil-Comp Press, pp. 117-129.

Lukacevic, M., Füssl, J., 2014. Numerical simulation tool for wooden boards with a physically based approach to identify structural failure. European Journal of Wood and Wood Products $72(4), 497-508$.

Lukacevic, M., Füssl, J., 2016. Application of a multisurface discrete crack model for clear wood taking into account the inherent microstructural characteristics of wood cells. Holzforschung $70(9), 845-853$.

Lukacevic, M., Füssl, J., Griessner, M., Eberhardsteiner, J., 2014a. Performance assessment of a numerical simulation tool for wooden boards with knots by means of full-field deformation measurements. Strain 50 (4), 301-317.

Lukacevic, M., Füssl, J., Lampert, R., 2014b. Failure mechanisms of clear wood identified at wood cell level by an approach based on the extended finite element mothod. Engineering fracture mechanics 144, 158-175.

Lukacevic, M., Lederer, W., Fssl, J., 2017. A microstructure-based multisurface failure criterion for the description of brittle and ductile failure mechanisms of clear-wood. Engineering Fracture Mechanics 176, 83 - 99 .

URL http://www.sciencedirect.com/science/article/pii/S0013794416307603

Lyamin, A., Sloan, S., 2000. Upper Bound Limit Analysis using Linear Finite Elements and Nonlinear Programming. Civil-Comp Press, pp. 131-145.

Lyamin, A. V., Sloan, S. W., October 2002a. Lower bound limit analysis using non-linear programming. International Journal for Numerical Methods in Engineering 55 (5), 573-611.

Lyamin, A. V., Sloan, S. W., 2002b. Upper bound limit analysis using linear finite elements and non-linear programming. International Journal for Numerical and Analytical Methods in Geomechanics2 26 (2), 181-216.

Lysmer, J., 1970. Limit analysis of plane problems in soil mechanics. Journal of the Soil Mechanics and Foundations Division 96 (4), 1311-1334. 
Mackenzie-Helnwein, P., Eberhardsteiner, J., Mang, H. A., 2003. A multi-surface plasticity model for clear wood and its application to the finite element analysis of structural details. Computational Mechanics 31 (1-2), 204-218.

Maier, G., Zavelani-Rossi, A., Benedetti, D., 1972. A finite element approach to optimal design of plastic structures in plane stress. International Journal for Numerical Methods in Engineering $4(4), 455-473$.

Makrodimopoulos, A., 2010. Remarks on some properties of conic yield restrictions in limit analysis. International Journal for Numerical Methods in Biomedical Engineering 26 (11), 1449-1461.

Makrodimopoulos, A., Martin, C., 2005a. Limit analysis using large-scale SOCP optimization. In: Proc. 13th Nat Conf. of UK Association for Computational Mechanics in Engineering. Sheffield, pp. 21-24.

Makrodimopoulos, A., Martin, C., 2005b. A novel formulation of upper bound limit analysis as a second-order cone programming problem. In: Proc. 8th International Conference on Computational Plasticity. Barcelona, pp. 1083-1086.

Makrodimopoulos, A., Martin, C. M., 2006. Lower bound limit analysis of cohesive-frictional materials using second-order cone programming. International Journal for Numerical Methods in Engineering 66 (4), 604-634.

Makrodimopoulos, A., Martin, C. M., 2007. Upper bound limit analysis using simplex strain elements and second-order cone programming. International Journal for Numerical and Analytical Methods in Geomechanics 31 (6), 835-865.

Makrodimopoulos, A., Martin, C. M., 2008. Upper bound limit analysis using discontinuous quadratic displacement fields. Communications in Numerical Methods in Engineering 24 (11), 911-927.

Masuda, M., 1988. Theoretical consideration on fracture criteria of wood - proposal of finite small area theory. In: Proceedings of the 1988 International Conference on Timber Engineering. Vol. 2. Seattle, pp. 584-595.

Michel, J., Moulinec, H., Suquet, P., 1999. Effective properties of composite materials with periodic microstructure: a computational approach. Computer Methods in Applied Mechanics and Engineering 172 (1), 109 - 143.

URL http://www.sciencedirect.com/science/article/pii/S0045782598002278

Milani, G., Loureno, P., Tralli, A., 2006a. Homogenised limit analysis of masonry walls, part i: Failure surfaces. Computers \& Structures 84 (3), 166 - 180.

Milani, G., Loureno, P., Tralli, A., 2006b. Homogenised limit analysis of masonry walls, part ii: Structural examples. Computers \& Structures 84 (3), 181 - 195. 
MOSEK ApS, 2014. The MOSEK optimization tools version 7.0. User's manual and reference, available from http://www.mosek.com [June 2014].

Pisano, A., Fuschi, P., Domenico, D. D., 2015. Numerical limit analysis of steel-reinforced concrete walls and slabs. Computers \& Structures 160, $42-55$.

Portioli, F., Casapulla, C., Gilbert, M., Cascini, L., 2014. Limit analysis of 3d masonry block structures with non-associative frictional joints using cone programming. Computers \& Structures $143,108-121$.

Saavedra Flores, E. I., Ajaj, R., Dayyani, I., Chandra, Y., Das, R., 2016. Multi-scale model updating for the mechanical properties of cross-laminated timber. Computers \& Structures 177, $83-90$.

URL http://www.sciencedirect.com/science/article/pii/S0045794916308574

Salmen, L., Burgert, I., 2009. Cell wall features with regard to mechanical performance. a review cost action E35 20042008: wood machining micromechanics and fracture. Holzforschung 63, $121-129$.

Schmidt, J., Kaliske, M., 2006. Zur dreidimensionalen Materialmodellierung von Fichtenholz mittels eines Mehrfächen-Plastizitätsmodells (in German). Holz als Roh- und Werkstoff 64 (5), 393-402.

Schmidt, J., Kaliske, M., 2007. Simulation of cracks in wood using a coupled material model for interface elements. Holzforschung 61 (4), 382-389.

Schmidt, J., Kaliske, M., 2009. Models for numerical failure analysis of wooden structures. Engineering Structures 31 (2), $571-579$.

Schwiedrzik, J., Raghavan, R., Rggeberg, M., Hansen, S., Wehrs, J., Adusumalli, R. B., Zimmermann, T., Michler, J., 2016. Identification of polymer matrix yield stress in the wood cell wall based on micropillar compression and micromechanical modelling. Philosophical Magazine $96(32-34), 3461-3478$.

URL http://dx.doi.org/10.1080/14786435.2016.1235292

Serrano, E., Gustafsson, P. J., 2007. Fracture mechanics in timber engineering strength analyses of components and joints. Materials and Structures 40 (1), 87-96.

Sinn, G., Reiterer, A., Stanzl-Tschegg, S. E., Tschegg, E. K., 2001. Determination of strain of thin wood samples using videoextensometry. Holz als Roh- und Werkstoff 59, 177-182.

Sjödin, J., Serrano, E., 2008. A numerical study of methods to predict the capacity of multiple steel-timber dowel joints. Holz als Roh- und Werkstoff 66 (6), 447-454.

Sjödin, J., Serrano, E., Enquist, B., 2008. An experimental and numerical study of the effect of friction in single dowel joints. Holz als Roh- und Werkstoff 66 (5), 363-372. 
802 Tsai, S. W., 1965. Strength characteristics of composite materials. Tech. rep., NASA.

Tsai, S. W., Wu, E. M., 1971. A general theory of strength for anisotropic materials. Journal of ${ }_{804}$ Composit Materials 5 (1), 58-80. 